\title{
ETROC1 Design Note
}

Abstract: This document provides the relevant information needed during ETROC1 design integration stage, and it is now improved to be used as a guide for testing ETROC1 prototype chips.

Version 1.0, September 23, 2020

Christopher Edwards ${ }^{a}$, Datao Gong ${ }^{b}$, Xing Huang ${ }^{b}$, Kent Liu ${ }^{b}$, Tiankuan Liu ${ }^{b}$, Ted Liua, Sandeep Miryalac, Jamieson Olsen ${ }^{a}$, Hanhan Sun ${ }^{b}$, Quan Sun ${ }^{a}$, Jinyuan $W_{u^{a}}$, Jingbo $\mathrm{Ye}^{\mathrm{b}}$, Li Zhang ${ }^{\mathrm{b}}$, Wei Zhang ${ }^{\mathrm{b}}$

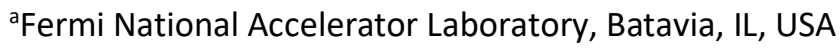

bSouthern Methodist University, Dallas, TX, USA

'Brookhaven National Laboratory, Upton, NY, USA

Technical contact: Quan Sun, qsun@fnal.gov

Project contact: Ted Liu, thliu@fnal.gov 


\section{FERMILAB-TM-2746-CMS-E}

Table of Contents

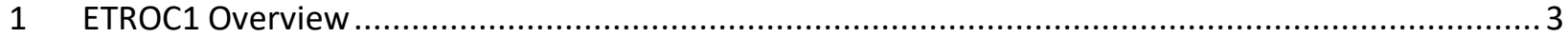

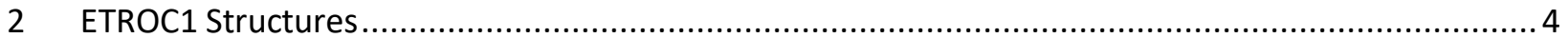

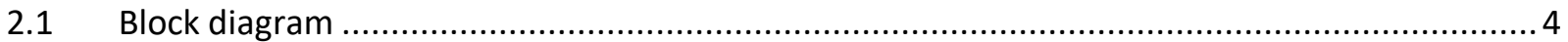

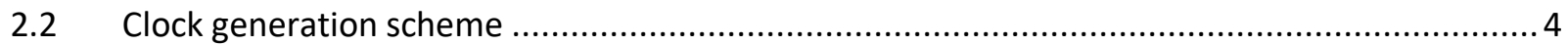

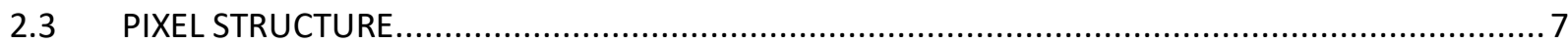

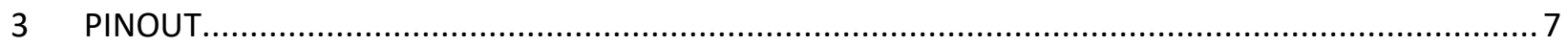

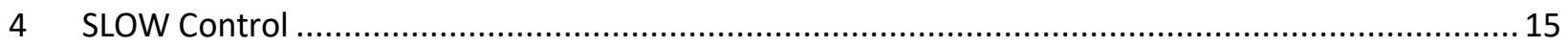

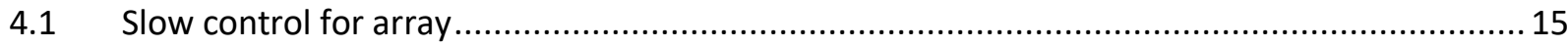

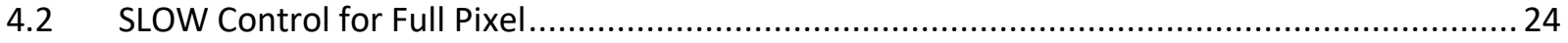

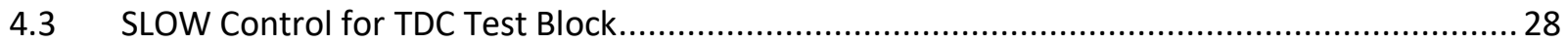

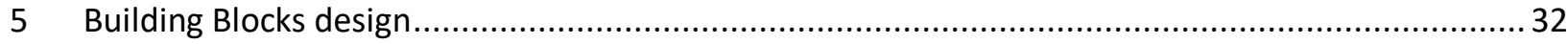

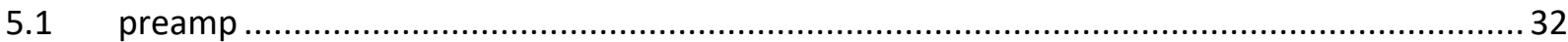

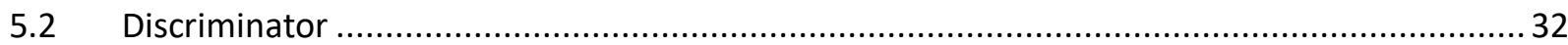

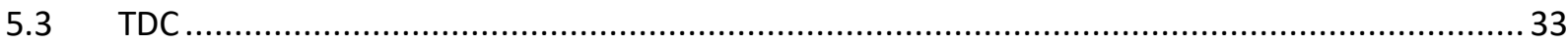

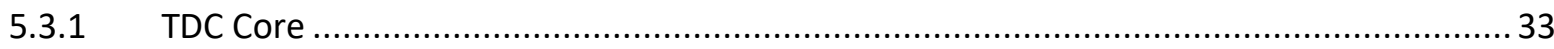

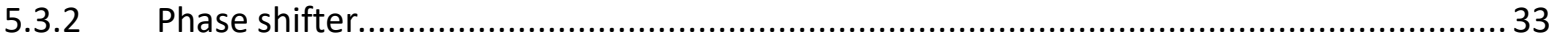

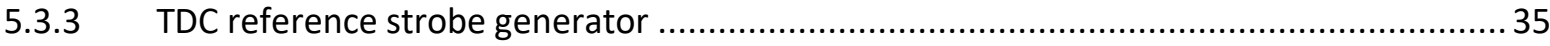

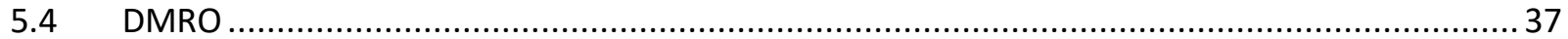

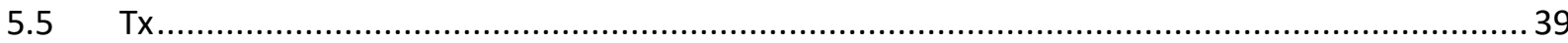

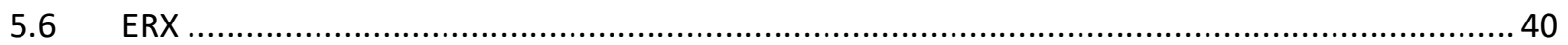

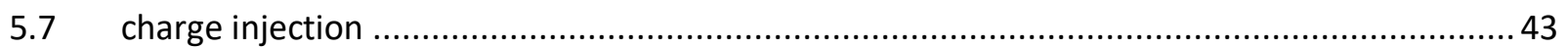

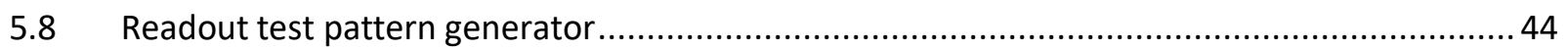

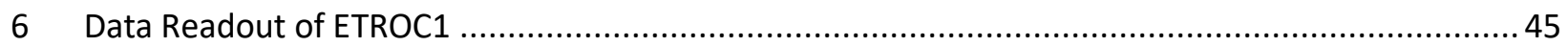

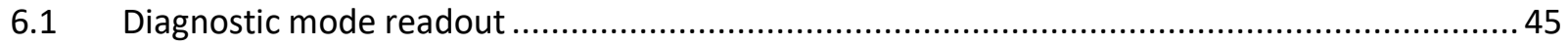

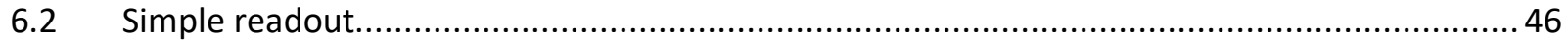




\section{ETROC1 OVERVIEW}

ETROC1 is the second prototype of ETROCn (ETL readout ASIC chip). It is developed with TSMC $65 \mathrm{~nm}$ CMOS process (CMN65, RF) and the metallization option is $1 \mathrm{p} 9 \mathrm{~m}-6 \times 1 \mathrm{z} 1 \mathrm{u}$.

ETROC1 aims to demonstrate full chain frontend, including the preamp, the discriminator and the TDC, and a $4 \times 4$ array. It interfaces with a $5 \times 5$ sensor with pixel size of $1.3 \times 1.3 \mathrm{~mm}^{2}$, which is similar to ALTIROC1. 17 ( 16 for the array and 1 for the standalone test block) out of the 25 pixels are to be connected to the readout circuits and other 8 pixels are dummies. Signal pads of dummy pixels are pulled down.

The tape-out date is Aug. 28, 2019, and turnaround is about 10 weeks. 


\section{ETROC1 STRUCTURES}

\subsection{BLOCK DIAGRAM}

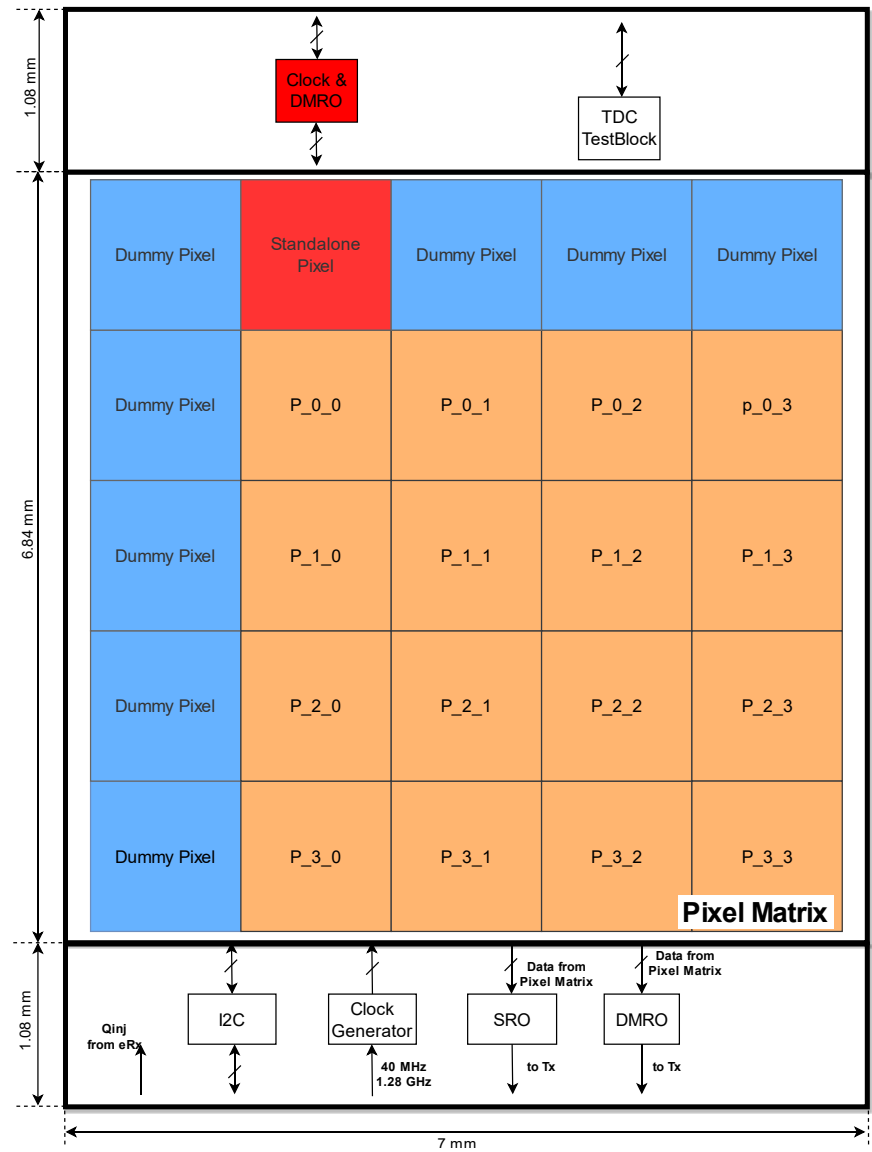

Figure 2.1.1 Block Diagram of ETROC1

\subsection{Clock Generation SCHEME}

The baseline clock generation scheme is receiving an off-chip $1.28 \mathrm{GHz}$ clock, generating the phaseshifted $40 \mathrm{MHz}$ and $320 \mathrm{MHz}$ clocks with the divider and the phase shifter. The TDC pulse generator generates pulses for the TDC and for off-chip checking. An optional scheme is directly receiving a 40 $\mathrm{MHz}$ clock and a $320 \mathrm{MHz}$ clock from the test board. 
FERMILAB-TM-2746-CMS-E

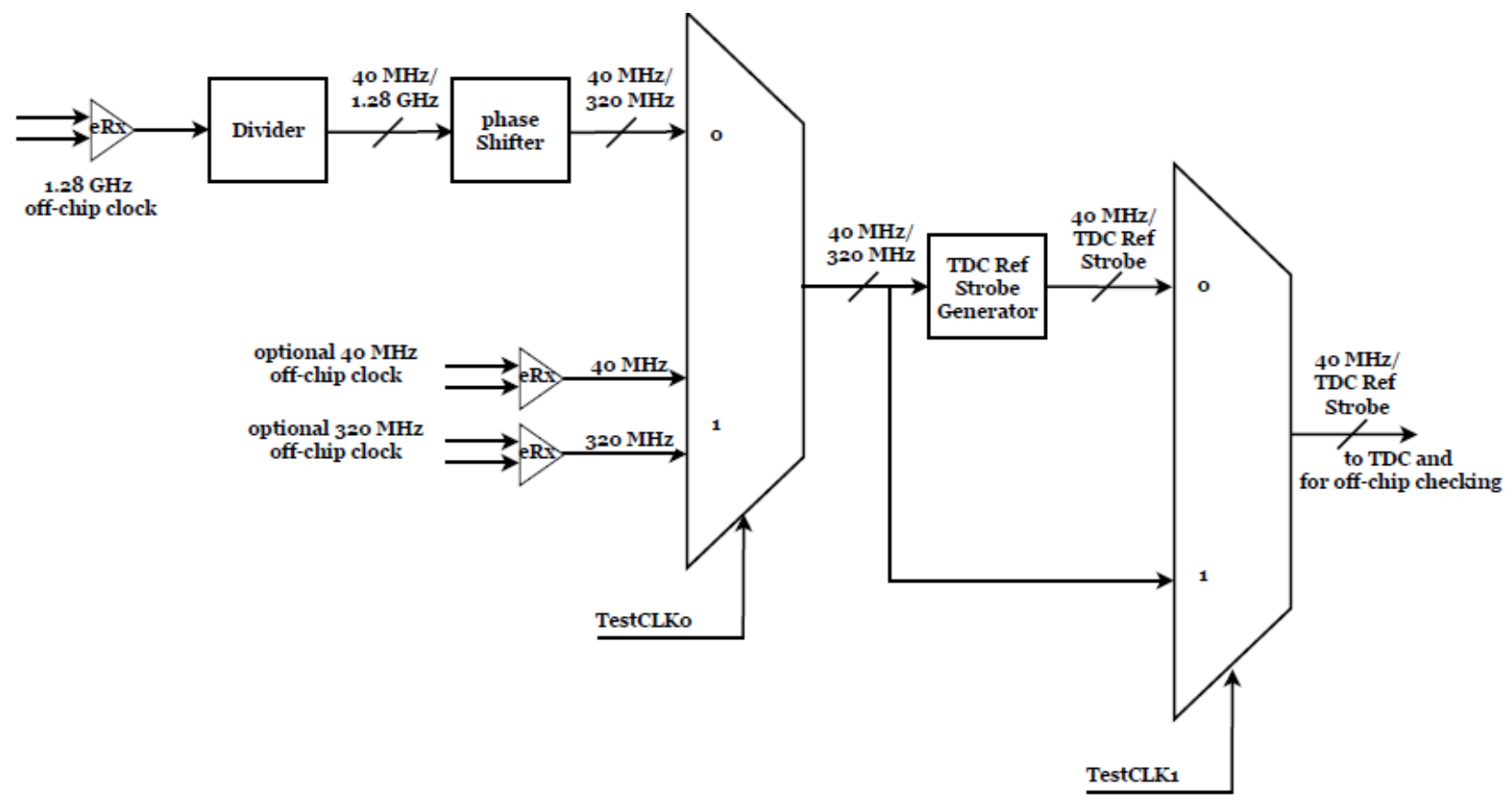

Figure 2.2.1 Block Diagram of the ETROC1 clock generator 


\section{FERMILAB-TM-2746-CMS-E}

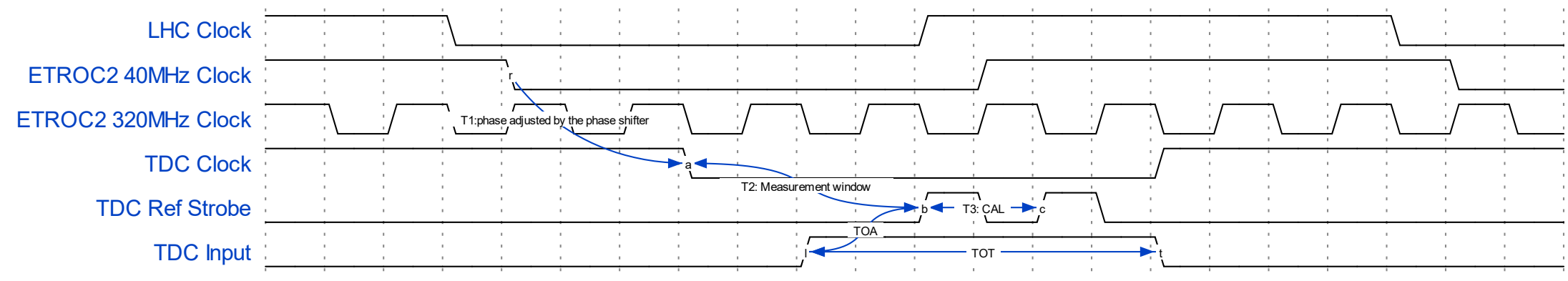

Figure 2.2.2 waveform of internal clock associated with TDC 


\subsection{PIXEL STRUCTURE}

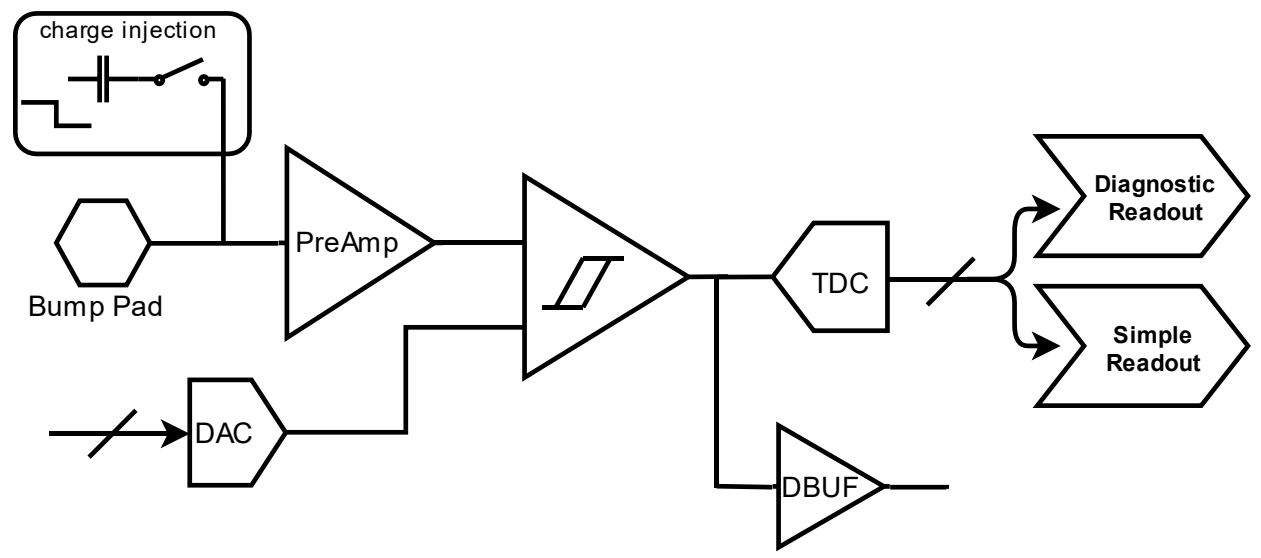

Figure 2.3.1 Pixel in array

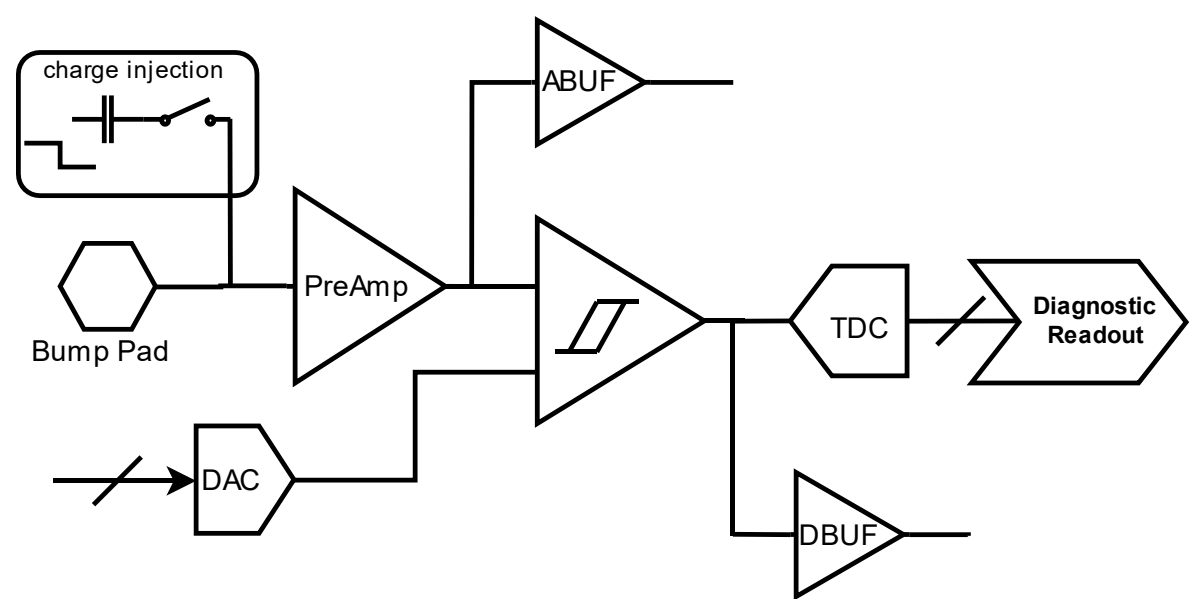

Figure 2.3.2 Standalone pixel

\section{PINOUT}

The suffixes, "_A", "_S" and "_T", represent the three blocks we have in ETROC1, array, standalone pixel and TDC, respectively. The opening of all the wire-bonding pads are $61 \mathrm{um}$ by $126 \mathrm{um}$.

Table 1 Wire bonding pad of the standalone pixel

\begin{tabular}{|l|l|l|l|l|}
\hline Num. & Name & Type & Description & Coordinate \\
\hline 1 & RSTN_S & Digital input & I2C Reset & $(4015.09,8872.21)$ \\
\hline
\end{tabular}




\begin{tabular}{|c|c|c|c|c|}
\hline 2 & SCL_S & Digital input & I2C clock & $(3905.09,8872.21)$ \\
\hline 3 & SDA_S & Digital input & $\mathrm{I} 2 \mathrm{C}$ data & $(3795.09,8872.21)$ \\
\hline 4 & CLKTO_N_S & \multirow[t]{2}{*}{$\begin{array}{l}\text { Differential } \\
\text { output }\end{array}$} & $\begin{array}{l}\text { Negative output of the test } \\
\text { clock, either } 40 \mathrm{MHz} \text { clock } \\
\text { or } 320 \mathrm{MHz} \text { pulse }\end{array}$ & $(3685.09,8872.21)$ \\
\hline 5 & CLKTO_P_S & & $\begin{array}{l}\text { Positive output of the test } \\
\text { clock, either } 40 \mathrm{MHz} \text { clock } \\
\text { or } 320 \mathrm{MHz} \text { pulse }\end{array}$ & $(3575.09,8872.21)$ \\
\hline 6 & vdd_Dig_S & Power & $\begin{array}{l}\text { Power supply of the digital } \\
\text { circuits, } 1.2 \mathrm{~V}\end{array}$ & $(3465.09,8872.21)$ \\
\hline 7 & vss_Dig_S & Ground & $\begin{array}{l}\text { Ground of the digital circuits, } \\
0 \mathrm{~V}\end{array}$ & $(3355.09,8872.21)$ \\
\hline 8 & DOut_N_S & \multirow[t]{2}{*}{$\begin{array}{l}\text { Differential } \\
\text { output }\end{array}$} & $\begin{array}{l}1.28 \text { Gbps data output of the } \\
\text { standalone pixel, negative }\end{array}$ & $(3245.09,8872.21)$ \\
\hline 9 & DOut_P_S & & $\begin{array}{l}1.28 \mathrm{Gbps} \text { data output of the } \\
\text { standalone pixel, positive }\end{array}$ & $(3135.09,8872.21)$ \\
\hline 10 & QInj_N_S & \multirow[t]{2}{*}{$\begin{array}{l}\text { Differential } \\
\text { input }\end{array}$} & $\begin{array}{l}\text { Negative input of the } \\
\text { charge injection trigger }\end{array}$ & $(3025.09,8872.21)$ \\
\hline 11 & QInj_P_S & & $\begin{array}{l}\text { Positive input of the charge } \\
\text { injection trigger }\end{array}$ & $(2915.09,8872.21)$ \\
\hline 12 & CLK40MI_N_S & \multirow[t]{2}{*}{$\begin{array}{l}\text { Differential } \\
\text { input }\end{array}$} & $\begin{array}{l}\text { Negative input of the } 40 \\
\text { MHz clock }\end{array}$ & $(2805.09,8872.21)$ \\
\hline 13 & CLK40MI_P_S & & $\begin{array}{l}\text { Positive input of the } 40 \\
\text { MHz clock }\end{array}$ & $(2695.09,8872.21)$ \\
\hline 14 & CLK320MI_N_S & \multirow[t]{2}{*}{$\begin{array}{l}\text { Differential } \\
\text { input }\end{array}$} & $\begin{array}{l}\text { Negative input of the } 320 \\
\text { MHz clock }\end{array}$ & $(2585.09,8872.21)$ \\
\hline 15 & CLK320MI_P_S & & $\begin{array}{l}\text { Positive input of the } 320 \\
\text { MHz clock }\end{array}$ & $(2475.09,8872.21)$ \\
\hline 16 & CLK1P28GI_N_S & $\begin{array}{l}\text { Differential } \\
\text { input }\end{array}$ & $\begin{array}{l}\text { Negative input of the } 1.28 \\
\text { GHz clock }\end{array}$ & $(2365.09,8872.21)$ \\
\hline
\end{tabular}




\begin{tabular}{|c|c|c|c|c|}
\hline 17 & CLK1P28GI_P_S & & $\begin{array}{l}\text { Positive input of the } 1.28 \\
\text { GHz clock }\end{array}$ & $(2255.09,8872.21)$ \\
\hline 18 & vdd_Dig_S & Power & $\begin{array}{l}\text { Power supply of the } \mathrm{Tx} \text { and } \\
\text { the } \mathrm{Rx}, 1.2 \mathrm{~V}\end{array}$ & $(2145.09,8872.21)$ \\
\hline 19 & vss_Dig_S & Ground & $\begin{array}{l}\text { Ground of the Tx and the Rx, } \\
0 \mathrm{~V}\end{array}$ & $(2035.09,8872.21)$ \\
\hline 20 & DiscriOut_S & Digital output & $\begin{array}{l}\text { Discriminator output of the } \\
\text { standalone pixel }\end{array}$ & $(1925.09,8872.21)$ \\
\hline 21 & PAIn_S & Analog input & Input of the preamp & $(1815.09,8872.21)$ \\
\hline 22 & PAIn_S & Analog input & Input of the preamp & $(1705.09,8872.21)$ \\
\hline 23 & vdd_PA_S & Power & $\begin{array}{l}\text { Power supply of the preamp, } \\
1.2 \mathrm{~V}\end{array}$ & $(1595.09,8872.21)$ \\
\hline 24 & vss_PA_S & Ground & Ground of the preamp, $0 \mathrm{~V}$ & $(1485.09,8872.21)$ \\
\hline 25 & VTHInOut_S & $\begin{array}{l}\text { Analog } \\
\text { output }\end{array}$ & DAC analog output & $(1375.09,8872.21)$ \\
\hline 26 & VRef_S & Analog input & $1 \mathrm{~V}$ reference input & $(1265.09,8872.21)$ \\
\hline 27 & AOut_S & Analog output & $\begin{array}{l}\text { Output of the analog buffer } \\
\text { in the standalone pixel }\end{array}$ & $(1155.09,8872.21)$ \\
\hline 28 & vdd_Buf_S & Power & $\begin{array}{l}\text { Power supply of the analog } \\
\text { buffer, } 1.5 \mathrm{~V}\end{array}$ & $(1045.09,8872.21)$ \\
\hline 29 & vss_Buf_S & Ground & $\begin{array}{l}\text { Ground of the analog buffer, } \\
0\end{array}$ & $(935.09,8872.21)$ \\
\hline 30 & QV_S & Analog inout & $\begin{array}{l}\text { Charge injection node } \\
\text { Can be used to monitor } \\
\text { voltage step size or to apply } \\
\text { an external negative step }\end{array}$ & $(825.09,8872.21)$ \\
\hline 31 & vdd_QInj_S & Power & $\begin{array}{l}\text { Power supply of the charge } \\
\text { injection, } 1.2 \mathrm{~V}\end{array}$ & $(715.09,8872.21)$ \\
\hline 32 & vss_QInj_S & Ground & $\begin{array}{l}\text { Ground of the charge } \\
\text { injection, } 0 \mathrm{~V}\end{array}$ & $(605.09,8872.21)$ \\
\hline
\end{tabular}


FERMILAB-TM-2746-CMS-E

\begin{tabular}{|l|l|l|l|l|}
\hline 33 & vdd_Discri_S & Power & $\begin{array}{l}\text { Power supply of the } \\
\text { discriminator, 1.2 V }\end{array}$ & $(495.09,8872.21)$ \\
\hline 34 & vss_Discri_S & Ground & $\begin{array}{l}\text { Ground of the discriminator, 0 } \\
\text { V }\end{array}$ & $(385.09,8872.21)$ \\
\hline
\end{tabular}

Table 2 Wire bonding pad of the array

\begin{tabular}{|c|c|c|c|c|}
\hline Num. & Name & Type & Description & Coordinate \\
\hline 1 & vdd_IO_A & Power & $\begin{array}{l}\text { Power supply of the } \mathrm{Tx} \text { and the } \mathrm{Rx} \text {, } \\
1.2 \mathrm{~V}\end{array}$ & $(1020,127.79)$ \\
\hline 2 & vss_IO_A & Ground & Ground of the Tx and the Rx, $0 \mathrm{~V}$ & $(1130,127.79)$ \\
\hline 3 & $\begin{array}{l}\text { CLK1P28GI_P } \\
\text { _A }\end{array}$ & \multirow[t]{2}{*}{$\begin{array}{l}\text { Differential } \\
\text { input }\end{array}$} & Positive input of the $1.28 \mathrm{GHz}$ clock & $(1240,127.79)$ \\
\hline 4 & $\begin{array}{l}\text { CLK1P28GI_N } \\
\text { _A }\end{array}$ & & Negative input of the $1.28 \mathrm{GHz}$ clock & $(1350,127.79)$ \\
\hline 5 & $\begin{array}{l}\text { CLK320MI_P } \\
\text { _A }\end{array}$ & \multirow[t]{2}{*}{$\begin{array}{l}\text { Differential } \\
\text { input }\end{array}$} & Positive input of the $320 \mathrm{MHz}$ clock & $(1460,127.79)$ \\
\hline 6 & $\begin{array}{l}\text { CLK320MI_N } \\
\text { _A }\end{array}$ & & Negative input of the $320 \mathrm{MHz}$ clock & $(1570,127.79)$ \\
\hline 7 & $\begin{array}{l}\text { CLK40MI_P } \\
\text { _A }\end{array}$ & \multirow[t]{2}{*}{$\begin{array}{l}\text { Differential } \\
\text { input }\end{array}$} & Positive input of the $40 \mathrm{MHz}$ clock & $(1680,127.79)$ \\
\hline 8 & $\begin{array}{l}\text { CLK40MI_N } \\
\text { _A }\end{array}$ & & Negative input of the $40 \mathrm{MHz}$ clock & $(1790,127.79)$ \\
\hline 9 & QInj_P_A & \multirow[t]{2}{*}{$\begin{array}{l}\text { Differential } \\
\text { input }\end{array}$} & $\begin{array}{l}\text { Positive input of the charge injection } \\
\text { trigger }\end{array}$ & $(1900,127.79)$ \\
\hline 10 & QInj_N_A & & $\begin{array}{l}\text { Negative input of the charge injection } \\
\text { trigger }\end{array}$ & $(2010,127.79)$ \\
\hline
\end{tabular}




\begin{tabular}{|c|c|c|c|c|}
\hline 11 & CLKTO_P_A & \multirow[t]{2}{*}{$\begin{array}{l}\text { Differential } \\
\text { output }\end{array}$} & $\begin{array}{l}\text { Positive output of the test clock, either } \\
40 \mathrm{MHz} \text { clock or } 320 \mathrm{MHz} \text { pulse }\end{array}$ & $(2120,127.79)$ \\
\hline 12 & CLKTO_N_A & & $\begin{array}{l}\text { Negative output of the test clock, } \\
\text { either } 40 \mathrm{MHz} \text { clock or } 320 \mathrm{MHz} \\
\text { pulse }\end{array}$ & $(2230,127.79)$ \\
\hline 13 & DOut_P_A & \multirow[t]{2}{*}{$\begin{array}{l}\text { Differential } \\
\text { output }\end{array}$} & $\begin{array}{l}\text { Positive data output of the array, for } \\
\text { either DMRO or SRO }\end{array}$ & $(2340,127.79)$ \\
\hline 14 & DOut_N_A & & $\begin{array}{l}\text { Negative data output of the array, for } \\
\text { either DMRO or SRO }\end{array}$ & $(2450,127.79)$ \\
\hline 15 & vdd_IO_A & Power & $\begin{array}{l}\text { Power supply of the } \mathrm{Tx} \text { and the } \mathrm{Rx} \text {, } \\
1.2 \mathrm{~V}\end{array}$ & $(2560,127.79)$ \\
\hline 16 & vss_IO_A & Ground & Ground of the Tx and the Rx, $0 \mathrm{~V}$ & $(2670,127.79)$ \\
\hline 17 & vdd_Discri_A & Power & $\begin{array}{l}\text { Power supply of the discriminator, } 1.2 \\
\text { V }\end{array}$ & $(2780,127.79)$ \\
\hline 18 & vss_Discri_A & Ground & Ground of the discriminator, $0 \mathrm{~V}$ & $(2890,127.79)$ \\
\hline 19 & vdd_PA_A & Power & Power supply of the preamp, $1.2 \mathrm{~V}$ & $(3000,127.79)$ \\
\hline 20 & vss_PA_A & Ground & Ground of the preamp, $0 \mathrm{~V}$ & $(3110,127.79)$ \\
\hline 21 & VRef_A & $\begin{array}{l}\text { Analog } \\
\text { input }\end{array}$ & $1 \mathrm{~V}$ reference input & $(3220,127.79)$ \\
\hline 22 & vdd_PA_A & Power & Power supply of the preamp, $1.2 \mathrm{~V}$ & $(3330,127.79)$ \\
\hline 23 & vss_PA_A & Ground & Ground of the preamp, $0 \mathrm{~V}$ & $(3440,127.79)$ \\
\hline 24 & VTHInOut_A & $\begin{array}{l}\text { Analog } \\
\text { Inout }\end{array}$ & $\begin{array}{l}\text { Threshold voltage of the } \\
\text { discriminators }\end{array}$ & $(3550,127.79)$ \\
\hline 25 & vdd_PA & Power & Power supply of the preamp, $1.2 \mathrm{~V}$ & $(3660,127.79)$ \\
\hline 26 & vss_PA_A & Ground & Ground of the preamp, $0 \mathrm{~V}$ & $(3770,127.79)$ \\
\hline 27 & vdd_QInj_A & Power & $\begin{array}{l}\text { Power supply of the charge injection, } \\
1.2 \mathrm{~V}\end{array}$ & $(3880,127.79)$ \\
\hline
\end{tabular}




\begin{tabular}{|c|c|c|c|c|}
\hline 28 & vss_QInj_A & Ground & Ground of the charge injection, $0 \mathrm{~V}$ & $(3990,127.79)$ \\
\hline 29 & vdd_CLK_A & Power & $\begin{array}{l}\text { Power supply of the clocks generation } \\
\text { circuits, } 1.2 \mathrm{~V}\end{array}$ & $(4100,127.79)$ \\
\hline 30 & vss_CLK_A & Ground & $\begin{array}{l}\text { Ground of the clocks generation } \\
\text { circuits, } 0 \mathrm{~V}\end{array}$ & $(4210,127.79)$ \\
\hline 31 & vdd_Discri_A & Power & $\begin{array}{l}\text { Power supply of the discriminator, } 1.2 \\
\text { V }\end{array}$ & $(4320,127.79)$ \\
\hline 32 & vss_Discri_A & Ground & Ground of the discriminator, $0 \mathrm{~V}$ & $(4430,127.79)$ \\
\hline 33 & vdd_Dig_A & Power & $\begin{array}{l}\text { Power supply of the digital circuits, } \\
1.2 \mathrm{~V}\end{array}$ & $(4540,127.79)$ \\
\hline 34 & vss_Dig_A & Ground & Ground of the digital circuits, $0 \mathrm{~V}$ & $(4650,127.79)$ \\
\hline 35 & BC0_A & $\begin{array}{l}\text { Digital } \\
\text { input }\end{array}$ & Bunch crossing zero & $(4760,127.79)$ \\
\hline 36 & L1ACC_A & $\begin{array}{l}\text { Digital } \\
\text { input }\end{array}$ & Level-1 acceptance & $(4870,127.79)$ \\
\hline 37 & RSTN_A & $\begin{array}{l}\text { Digital } \\
\text { input }\end{array}$ & $\begin{array}{l}\text { Reset for I2C/SRO Controller/DMRO } \\
\text { internally pulled up }\end{array}$ & $(4980,127.79)$ \\
\hline 38 & DiscriOut_A & $\begin{array}{l}\text { Digital } \\
\text { output }\end{array}$ & Discriminator output of the array & $(5090,127.79)$ \\
\hline 39 & A0_A & $\begin{array}{l}\text { Digital } \\
\text { input }\end{array}$ & I2C address low bit for the slave $\mathrm{A}$ & $(5200,127.79)$ \\
\hline 40 & A1_A & $\begin{array}{l}\text { Digital } \\
\text { input }\end{array}$ & I2C address high bit for the slave $\mathrm{B}$ & $(5310,127.79)$ \\
\hline 41 & vdd_Dig_A & Power & $\begin{array}{l}\text { Power supply of the digital circuits, } \\
1.2 \mathrm{~V}\end{array}$ & $(5420,127.79)$ \\
\hline 42 & vss_Dig_A & Ground & Ground of the digital circuits, $0 \mathrm{~V}$ & $(5530,127.79)$ \\
\hline 43 & SCL_A_A & $\begin{array}{l}\text { Digital } \\
\text { input }\end{array}$ & I2C clock for the slave A & $(5640,127.79)$ \\
\hline 44 & SDA_A_A & $\begin{array}{l}\text { Digital } \\
\text { input }\end{array}$ & I2C data for the slave $\mathrm{A}$ & $(5750,127.79)$ \\
\hline
\end{tabular}




\begin{tabular}{|l|l|l|l|l|}
\hline 45 & A0_B_A & $\begin{array}{l}\text { Digital } \\
\text { input }\end{array}$ & I2C address low bit for the slave A & $(5860,127.79)$ \\
\hline 46 & A1_B_A & $\begin{array}{l}\text { Digital } \\
\text { input }\end{array}$ & I2C address high bit for the slave B & $(5970,127.79)$ \\
\hline 47 & SCL_B_A & $\begin{array}{l}\text { Digital } \\
\text { input }\end{array}$ & I2C clock for the slave B & $(6080,127.79)$ \\
\hline 48 & SDA_B_A & $\begin{array}{l}\text { Digital } \\
\text { input }\end{array}$ & I2C data for the slave B & $(6190,127.79)$ \\
\hline 49 & vdd_Dig_A & Power & $\begin{array}{l}\text { Power supply of the digital circuits, } \\
1.2 \mathrm{~V}\end{array}$ & $(6300,127.79)$ \\
\hline 50 & vss_Dig_A & Ground & Ground of the digital circuits, 0 V & $(6410,127.79)$ \\
\hline
\end{tabular}

Table 3 pinout of the TDC test block

\begin{tabular}{|l|l|l|l|c|}
\hline Num. & Name & Type & Description & Coordinate \\
\hline 1 & DOut_N_T & $\begin{array}{l}\text { Digital } \\
\text { Out }\end{array}$ & $\begin{array}{l}1.28 \mathrm{G} \text { high speed serial data } \\
\text { negative output }\end{array}$ & $(6872.21,8373.85)$ \\
\hline 2 & DOut_P_T & $\begin{array}{l}\text { Digital } \\
\text { Out }\end{array}$ & $\begin{array}{l}1.28 \mathrm{G} \text { high speed serial data } \\
\text { positive output }\end{array}$ & $(6872.21,8450.03)$ \\
\hline 3 & DMRO_vdd_T & $\begin{array}{l}\text { Power } \\
\text { supply }\end{array}$ & $\begin{array}{l}\text { Power Supply of DMRO } \\
\text { module, 1.2V }\end{array}$ & $(6872.21,8526.21)$ \\
\hline 4 & DMRO_vss_T & Ground & Ground of the DMRO module & $(6872.21,8602.39)$ \\
\hline 5 & CLK40MO_N_T & $\begin{array}{l}\text { Digital } \\
\text { Out }\end{array}$ & 40M clock negative output & $(6872.21,8678.57)$ \\
\hline 6 & CLK40MO_P_T & $\begin{array}{l}\text { Digital } \\
\text { Out }\end{array}$ & 40M clock positive output & $(6872.21,8754.75)$ \\
\hline
\end{tabular}




\begin{tabular}{|c|c|c|c|c|}
\hline 7 & I2C_vdd_T & $\begin{array}{l}\text { Power } \\
\text { Supply }\end{array}$ & $\begin{array}{l}\text { Power supply of I2C module } \\
\text { and Clock modules, } 1.2 \mathrm{~V}\end{array}$ & $(6720.68,8872.21)$ \\
\hline 8 & I2C_vss_T & Ground & $\begin{array}{l}\text { Ground of I2C module and } \\
\text { Clock modules }\end{array}$ & $(6644.50,8872.21)$ \\
\hline 9 & CLK1P28GI_P_T & $\begin{array}{l}\text { Digital } \\
\text { In }\end{array}$ & 1.28G clock positive input & $(6563.92,8872.21)$ \\
\hline 10 & CLK1P28GI_N_T & $\begin{array}{l}\text { Digital } \\
\text { In }\end{array}$ & $1.28 \mathrm{G}$ clock negative input & $(6483.34,8872.21)$ \\
\hline 11 & CLK320MI_P_T & $\begin{array}{l}\text { Digital } \\
\text { In }\end{array}$ & $320 \mathrm{M}$ clock positive input & $(6402.76,8872.21)$ \\
\hline 12 & CLK320MI_N_T & $\begin{array}{l}\text { Digital } \\
\text { In }\end{array}$ & $320 \mathrm{M}$ clock negative input & $(6322.18,8872.21)$ \\
\hline 13 & CLK40MI_P_T & $\begin{array}{l}\text { Digital } \\
\text { In }\end{array}$ & 40M clock positive input & $(6241.61,8872.21)$ \\
\hline 14 & CLK40MI_N_T & $\begin{array}{l}\text { Digital } \\
\text { In }\end{array}$ & 40M clock negative input & $(6161.03,8872.21)$ \\
\hline 15 & Pulse_P_T & $\begin{array}{l}\text { Analog } \\
\text { In }\end{array}$ & Pulse positive input & $(6080.45,8872.21)$ \\
\hline 16 & Pulse_N_T & $\begin{array}{l}\text { Analog } \\
\text { In }\end{array}$ & Pulse negative input & $(5999.87,8872.21)$ \\
\hline 17 & TDC_vss_T & Ground & Ground of the TDC module & $(5919.29,8872.21)$ \\
\hline 18 & TDC_vdd_T & $\begin{array}{l}\text { Power } \\
\text { supply }\end{array}$ & $\begin{array}{l}\text { Power supply of the TDC } \\
\text { module, } 1.2 \mathrm{~V}\end{array}$ & $(5843.11,8872.21)$ \\
\hline 19 & SCL_T & $\begin{array}{l}\text { Digital } \\
\text { In }\end{array}$ & I2C module serial clock & $(5756.33,8872.21)$ \\
\hline 20 & SDA_T & $\begin{array}{l}\text { Digital } \\
\text { Out/In }\end{array}$ & I2C module serial data & $(5651.26,8872.21)$ \\
\hline 21 & RSTN_T & $\begin{array}{l}\text { Digital } \\
\text { In }\end{array}$ & I $2 \mathrm{C}$ module reset, low active & $(5546.18,8872.21)$ \\
\hline 22 & A0_T & $\begin{array}{l}\text { Digital } \\
\text { In }\end{array}$ & $\begin{array}{l}\text { I2C module external address } \\
\text { select }\end{array}$ & $(5441.11,8872.21)$ \\
\hline
\end{tabular}


FERMILAB-TM-2746-CMS-E

\begin{tabular}{|l|l|l|l|l|}
\hline 23 & vss_GRO_T & Ground & $\begin{array}{l}\text { Ground of Gate Ring Oscillator } \\
\text { module. }\end{array}$ & $(5350.91,8872.21)$ \\
\hline 24 & vdd_GRO_T & $\begin{array}{l}\text { Power } \\
\text { Suppl }\end{array}$ & $\begin{array}{l}\text { Power supply of Gate Ring } \\
\text { Oscillator module. }\end{array}$ & $(5274.73$ 8872.21) \\
\hline 25 & GRO_Out_P_T & $\begin{array}{l}\text { Digital } \\
\text { Out }\end{array}$ & $\begin{array}{l}\text { Gate Ring Oscillator positive } \\
\text { output }\end{array}$ & $(5198.55,8872.21)$ \\
\hline 26 & GRO_Out_N_T & $\begin{array}{l}\text { Digital } \\
\text { Out }\end{array}$ & $\begin{array}{l}\text { Gate Ring Oscillator negative } \\
\text { output }\end{array}$ & $(5122.37,8872.21)$ \\
\hline
\end{tabular}

\section{SLOW CONTROL}

\subsection{SLOW CONTROL FOR ARRAY}

A generic $12 \mathrm{C}$ slave is used twice in ETROC1. Each slave provides 32 bytes for reading and 16 bytes for writing by ETROC1. A 4-bit chip ID and a 4-bit chip reversion are available as well. The registers in the I2C slave are triplicated to mitigate SEU. The registers in two slaves are named REG_A and REG_B,

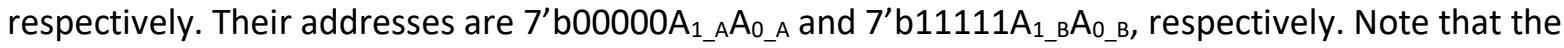
address of 7 'b0000000 should be avoided for the slave $A$.

\begin{tabular}{|c|c|c|c|c|}
\hline Num. & REG. NAME & $\begin{array}{l}\text { REG. } \\
\text { ADDR. }\end{array}$ & Description & Default \\
\hline 1 & CLSel[1:0] & REG_A_00[1:0] & $\begin{array}{l}\text { Select of load capacitance of } \\
\text { the preamp first stage } \\
2^{`} \text { b00--> } 0 \mathrm{fC} \\
2^{`} \text { b01--> } 80 \mathrm{fC} \\
2^{`} \mathrm{~b} 10-->80 \mathrm{fC} \\
2^{`} \text { b01--> } 160 \mathrm{fC} \\
\text { Shared by all the pixels }\end{array}$ & 2'b00 \\
\hline 2 & DIS_VTHInOut[15:0] ${ }^{a}$ & $\begin{array}{l}\text { REG_A_03, } \\
\text { REG_A_02 }\end{array}$ & $\begin{array}{l}\text { Disable threshold voltage } \\
\text { input/output of the specified } \\
\text { pixel. } \\
\text { Active high. Each bit controls a } \\
\text { pixel according the pixels index } \\
\text { map. } \\
\text { Only one of thresholds can be } \\
\text { enabled at a time. } \\
\text { For example, } \\
\text { DIS_VTHInOut = } 16^{\prime} \text { h4000 }\end{array}$ & $16^{\prime}$ hffff \\
\hline
\end{tabular}


FERMILAB-TM-2746-CMS-E

\begin{tabular}{|c|c|c|c|c|}
\hline 3 & EN_DiscriOut[7:0] & REG_A_04 & $\begin{array}{l}\text { Enable the discriminator } \\
\text { output. } \\
\text { Active high. } \\
\text { Each bit in EN_DiscriOut[7:4] } \\
\text { represents the row, and each } \\
\text { bit in EN_DiscriOut[3:0] } \\
\text { represents the column. Users } \\
\text { can enable the discriminator } \\
\text { output from a specified pixel. } \\
\text { Only one row can be specified } \\
\text { at a time. That means no more } \\
\text { than one bit in } \\
\text { EN_DiscriOut[7:4] can be set to } \\
\text { 1'b1 at a time. } \\
\text { When more than one bit is set } \\
\text { to 1'b1, or all bits are set to } 0 \text { in } \\
\text { EN_DiscriOut[3:0], the } \\
\text { discriminator output is } \\
\text { disabled. } \\
\text { For example: } \\
\text { 8'b0010_0100 } \rightarrow \text { pixel in row } 1 \\
\text { and column } 2 \\
\text { 8'b0001_0001 } \rightarrow \text { pixel in row } 0 \\
\text { and column } 0 \\
\text { 8'b1000_0100 } \rightarrow \text { pixel in row } 3 \\
\text { and column } 2 \\
\text { 8'bxxxx_0101 } \rightarrow \text { disable } \\
\text { discriminator output, but } \\
\text { invalid } \\
\text { 8'b1011_xxxx } \rightarrow \text { invalid } \\
\text { 8'b0000_0111 } \rightarrow \text { disable } \\
\text { discriminator output } \\
\text { 8'b0000_0000 } \rightarrow \text { disable } \\
\text { discriminator output } \\
\text { and }\end{array}$ & 8'b00010001 \\
\hline 4 & EN_QInj[15:0] $]^{a}$ & $\begin{array}{l}\text { REG_A_06, } \\
\text { REG_A_05 }\end{array}$ & $\begin{array}{l}\text { enable the charge injection of } \\
\text { the specified pixel. } \\
\text { Active high. Each bit controls a } \\
\text { pixel. } \\
\text { Users can specify non or more } \\
\text { pixels to enable the charge } \\
\text { injection. }\end{array}$ & $16^{\prime}$ h0001 \\
\hline 5 & HysSel[3:0] & $\begin{array}{l}\text { REG_A } \\
\text { _00[7:4] }\end{array}$ & $\begin{array}{l}\text { Hysteresis voltage selection } \\
\text { 4`b0000 --> Vhys1 } \\
\text { 4`b0001 --> Vhys2 } \\
\text { 4`b0011 --> Vhys3 }\end{array}$ & 4’b1111 \\
\hline
\end{tabular}




\begin{tabular}{|c|c|c|c|c|}
\hline & & & $\begin{array}{l}\text { 4`b0111 --> Vhys4 } \\
\text { 4`b1111 --> Vhys5 } \\
\text { Vhys1 > Vhys2 > Vhys3 > Vhys4 } \\
=\text { Vhys5 = } 0 \\
\text { Shared by all the pixels }\end{array}$ & \\
\hline 6 & IBSel[2:0] & $\begin{array}{l}\text { REG_A } \\
\text { 01[2:0] }\end{array}$ & $\begin{array}{l}\text { Bias current selection of the } \\
\text { input transistor in the preamp } \\
3^{`} b 000-->11 \\
3^{`} b 001,3^{`} b 010,3^{`} b 100-->12 \\
3^{`} b 011,3^{`} b 110,3^{`} b 101-->13 \\
3^{`} b 111-->14 \\
11>12>13>14 \\
\text { Shared by all the pixels }\end{array}$ & 3`b111 \\
\hline 7 & OE_DMRO_Row[3:0] & $\begin{array}{l}\text { REG_A } \\
\_07[3: 0]\end{array}$ & $\begin{array}{l}\text { Output enable of DMRO in } \\
\text { rows. Each bit represents a } \\
\text { row. Only one row can be } \\
\text { enabled for output at a time. } \\
\text { For example: } \\
\text { 4'b0000 } \rightarrow \text { no DMRO output } \\
\text { enabled } \\
\text { 4'b0001 } \rightarrow \text { the row } 0 \text { of DMRO } \\
\text { output is enabled } \\
\text { 4'b0100 } \rightarrow \text { the row } 2 \text { of DMRO } \\
\text { output is enable } \\
4^{\prime} \text { b1010 } \rightarrow \text { invalid }\end{array}$ & 4'b0001 \\
\hline 8 & DMRO_COL[1:0] & $\begin{array}{l}\text { REG_A } \\
\_07[5: 4]\end{array}$ & $\begin{array}{l}\text { Select DMRO output from a } \\
\text { specified column. } \\
\text { 4'b00 } \rightarrow \text { column } 0 \\
4^{\prime} \text { b0 } 1 \rightarrow \text { column } 1 \\
4^{\prime} \text { b10 } \rightarrow \text { column } 2 \\
4^{\prime} \text { b11 } \rightarrow \text { column } 3\end{array}$ & 2'b00 \\
\hline 9 & RO_SEL & REG_A_07[6] & $\begin{array}{l}\text { Select readout mode from } \\
\text { either SRO or DMRO. } \\
1^{\prime} b 0 \rightarrow \text { DMRO enabled } \\
1^{\prime} b 1 \rightarrow \text { SRO enabled }\end{array}$ & 1'b0 \\
\hline 10 & PD_DACDiscri[15:0] & $\begin{array}{l}\text { REG_A_09, } \\
\text { REG_A_08 }\end{array}$ & $\begin{array}{l}\text { Power down the DAC and the } \\
\text { discriminator in pixels. } \\
\text { Active high. Each bit controls a } \\
\text { pixel. } \\
\text { Users can specify non or more } \\
\text { pixels to control. }\end{array}$ & $16^{\prime}$ h0000 \\
\hline 11 & QSel<4:0> & $\begin{array}{l}\text { REG_A } \\
\text { _01[7:3] }\end{array}$ & $\begin{array}{l}\text { Select injected charge, from } 1 \\
\mathrm{fC}\left(5^{`} \text { b00000) to } 32\right. \\
\mathrm{fC}\left(5^{`} \mathrm{~b} 11111\right)\end{array}$ & $5^{\prime} \mathrm{b} 00110$ \\
\hline
\end{tabular}


FERMILAB-TM-2746-CMS-E

\begin{tabular}{|c|c|c|c|c|}
\hline & & & $\begin{array}{l}\text { Typical charge from LGAD } \\
\text { sensor is } 7 \mathrm{fC}\left(5^{\prime} \text { 'b00110) }\right. \\
\text { Shared by all the pixels }\end{array}$ & \\
\hline 12 & RfSel[1:0] & $\begin{array}{l}\text { REG_A } \\
\_00[3: 2]\end{array}$ & $\begin{array}{l}\text { Feedback resistance selection } \\
\text { 2`b00--> } 20 \mathrm{kOHm} \\
\text { 2`b01--> } 10 \mathrm{kOHm} \\
\text { 2`b10--> } 5.7 \mathrm{kOHm} \\
\text { 2`b11--> } 4.4 \mathrm{kOHm} \\
\text { Shared by all the pixels }\end{array}$ & 2`b10 \\
\hline 13 & VTHIn[159:0] & $\begin{array}{l}\text { REG_A_0A, } \\
\text { REG_A_0B, } \\
\text { REG_A_0C, } \\
\text { REG_A_0D, } \\
\text { REG_A_0E, } \\
\text { REG_A_0F, } \\
\text { REG_A_10, } \\
\text { REG_A_11, } \\
\text { REG_A_12, } \\
\text { REG_A_13, } \\
\text { REG_A_14, } \\
\text { REG_A_15, } \\
\text { REG_A_16, } \\
\text { REG_A_17, } \\
\text { REG_A_18, } \\
\text { REG_A_19, } \\
\text { REG_A_1A, } \\
\text { REG_A_1B, } \\
\text { REG_A_1C, } \\
\text { REG_A_1D }\end{array}$ & $\begin{array}{l}\text { Threshold voltage input of all } \\
\text { the } 16 \text { pixels } \\
\text { VTHIn[159:0] } \rightarrow\{\text { VTHIn15, } \\
\text { VTHIn14, ..., VTHIn1, VTHIn0\} } \\
\text { VTHIn15 represents DAC } \\
\text { setting in pixel 15... }\end{array}$ & $\begin{array}{l}\text { For each DAC, } \\
\text { the default value } \\
\text { is } \\
10^{\prime} b 1000000000\end{array}$ \\
\hline 14 & autoReset_TDC & REG_B_00[0] & & 1'b0 \\
\hline 15 & enableMon_TDC & REG_B_00[1] & $\begin{array}{l}\text { This bit is actually used to } \\
\text { control the readout test mode } \\
\text { in ROTestGen. }\end{array}$ & 1'b0 \\
\hline 16 & enable_TDC & REG_B_00[2] & & 1'b1 \\
\hline 17 & level_TDC[2:0] & REG_B_01[2:0] & & 3'b001 \\
\hline 18 & offset_TDC[6:0] & REG_B_02[6:0] & & 7'b0000000 \\
\hline 19 & polaritySel_TDC & REG_B_00[3] & & 1'b1 \\
\hline 20 & resetn_TDC & REG_B_00[4] & & 1'b1 \\
\hline 21 & selRawCode_TDC & REG_B_00[5] & & 1'b0 \\
\hline 22 & testMode_TDC & REG_B_00[6] & & 1'b0 \\
\hline 23 & timeStampMode_TDC & REG_B_00[7] & & 1'b0 \\
\hline 24 & ROI[15:0] & $\begin{array}{l}\text { REG_A_1F, } \\
\text { REG_A_1E }\end{array}$ & $\begin{array}{l}\text { Region of interest. } \\
\text { 16-bit vector specifies which } \\
\text { pixels are enabled for readout. }\end{array}$ & $16^{\prime} \mathrm{hffff}$ \\
\hline
\end{tabular}


FERMILAB-TM-2746-CMS-E

\begin{tabular}{|c|c|c|c|c|}
\hline 25 & dllEnable & REG_B_03[0] & $\begin{array}{l}\text { Enable loop control of DLL. The } \\
\text { control voltage is tied to } \\
\text { ground when dllEnable==low. }\end{array}$ & 1'b1 \\
\hline 26 & dllForceDown & REG_B_03[1] & $\begin{array}{l}\text { Force to pull down the output } \\
\text { of the phase detector, active } \\
\text { high. }\end{array}$ & 1'b0 \\
\hline 27 & dllCapReset & REG_B_03[2] & $\begin{array}{l}\text { Reset the control voltage of DLL } \\
\text { to power supply, active high. }\end{array}$ & $1^{\prime} \mathrm{b} 0$ \\
\hline 28 & dIICPCurrent[3:0] & REG_B_03[6:3] & $\begin{array}{l}\text { Charge pump current control } \\
\text { bits, ranging from } 0 \text { to } 15 \mathrm{uA} \text { for } \\
\text { charge and discharge. }\end{array}$ & 4'b0001 \\
\hline 29 & dllLate & REG_A_20[0] & Lock status prompt. & $\begin{array}{l}\text { Read from } \\
\text { ETROC1 }\end{array}$ \\
\hline 30 & PhaseAdj[7:0] & REG_B_04 & $\begin{array}{l}\text { Phase selecting control bits, } \\
\text { PhaseAdj }<7: 3>\text { for coarse, } \\
\text { PhaseAdj }<2: 0>\text { for fine. }\end{array}$ & 8'b00000000 \\
\hline 31 & RefStrSel[7:0] & REG_B_05 & TDC reference strobe selection. & 8'b00000011, \\
\hline 32 & ENScr_DMRO & REG_B_06[0] & Enable scrambling, active high & 1'b1 \\
\hline 33 & REVCLK_DMRO & REG_B_06[1] & $\begin{array}{l}\text { Reversing the clock used for } \\
\text { input data latch, active-high. } \\
\text { When REVData=0 data is } \\
\text { latched at the rising edges of } \\
\text { CLKWord, otherwise data is } \\
\text { latched at the falling edges of } \\
\text { CLKWord. }\end{array}$ & 1'b0 \\
\hline 34 & REVData_DMRO & REG_B_06[2] & $\begin{array}{l}\text { reversing input data, active- } \\
\text { high. }\end{array}$ & 1'b0 \\
\hline 35 & TestMode_DMRO & REG_B_06[3] & $\begin{array}{l}\text { Test mode input, active high. } \\
\text { The PRBS7 is sent out in test } \\
\text { mode (TestMode }==1 \text { ) while } \\
\text { the data is sent out in normal } \\
\text { mode (TestMode ==1). }\end{array}$ & 1'b0 \\
\hline 36 & TestCLKO & REG_B_06[4] & $\begin{array}{l}\text { When TestCLKO }=1 \text {, the phase } \\
\text { shifter is bypassed and off-chip } \\
40 \mathrm{MHz} \text { and } 320 \mathrm{MHz} \text { are used. }\end{array}$ & 1'b0 \\
\hline 37 & TestCLK1 & REG_B_06[5] & $\begin{array}{l}\text { When TestCLK } 1=1 \text {, the TDC } \\
\text { reference strobe generator is } \\
\text { bypassed and off-chip } 40 \mathrm{MHz} \\
\text { and } 320 \mathrm{MHz} \text { are used. }\end{array}$ & 1'b0 \\
\hline 38 & CLKOutSel & REG_B_06[6] & $\begin{array}{l}\text { Select output from either } 40 \\
\text { MHz clock or TDC reference } \\
\text { strobe } \\
\text { 1'b0: } 40 \mathrm{MHz} \text { clock } \\
\text { 1'b1: TDC reference strobe }\end{array}$ & 1'b1 \\
\hline
\end{tabular}


FERMILAB-TM-2746-CMS-E

\begin{tabular}{|c|c|c|c|c|}
\hline 39 & enableRx_1P28G & REG_B_07[5] & $\begin{array}{l}\text { Enable the Rx for } 1.28 \mathrm{GHz} \\
\text { clock, active high }\end{array}$ & 1'b1 \\
\hline 40 & setCM_1P28G & REG_B_07[4] & $\begin{array}{l}\text { Set common voltage of the Rx } \\
\text { for the } 1.28 \mathrm{GHz} \text { clock to } 1 / 2 \mathrm{vdd} \text {, } \\
\text { active high }\end{array}$ & 1'b1 \\
\hline 41 & enableTER_1P28G & REG_B_07[3] & $\begin{array}{l}\text { Enable internal termination of } \\
\text { the Rx for the } 1.28 \mathrm{GHz} \text { clock, } \\
\text { active high }\end{array}$ & 1'b1 \\
\hline 42 & invertData_1P28G & REG_B_07[2] & $\begin{array}{l}\text { Invert data of the Rx for the } \\
1.28 \mathrm{GHz} \text { clock, active high }\end{array}$ & 1'b0 \\
\hline 43 & equ_1P28G[1:0] & REG_B_07[1:0] & $\begin{array}{l}\text { Equalization strength of the Rx } \\
\text { for the } 320 \mathrm{MHz} \text { clock } \\
\text { 2'b00, equalization is turned off } \\
\text { 2'b11, largest equalization }\end{array}$ & 2'b00 \\
\hline 44 & enableRx_320M & REG_B_08[5] & $\begin{array}{l}\text { Enable the Rx for the } 320 \mathrm{MHz} \\
\text { clock, active high }\end{array}$ & 1'b0 \\
\hline 45 & setCM_320M & REG_B_08[4] & $\begin{array}{l}\text { Set common voltage of the Rx } \\
\text { for the } 320 \mathrm{MHz} \text { clock to } 1 / 2 \mathrm{vdd} \text {, } \\
\text { active high }\end{array}$ & 1'b1 \\
\hline 46 & enableTER_320M & REG_B_08[3] & $\begin{array}{l}\text { Enable internal termination of } \\
\text { the Rx for the } 320 \mathrm{MHz} \text { clock, } \\
\text { active high }\end{array}$ & 1'b1 \\
\hline 47 & invertData_320M & REG_B_08[2] & $\begin{array}{l}\text { Invert data of the Rx for the } \\
320 \mathrm{MHz} \text { clock, active high }\end{array}$ & 1'b0 \\
\hline 48 & equ_320M [1:0] & REG_B_08[1:0] & $\begin{array}{l}\text { Equalization strength of the Rx } \\
\text { for the } 320 \mathrm{MHz} \text { clock } \\
\text { 2'b00, equalization is turned off } \\
\text { 2'b11, largest equalization }\end{array}$ & 2'b00 \\
\hline 49 & enableRx_40M & REG_B_09[5] & $\begin{array}{l}\text { Enable the Rx for the } 40 \mathrm{MHz} \\
\text { clock, active high }\end{array}$ & 1'b0 \\
\hline 50 & setCM_40M & REG_B_09[4] & $\begin{array}{l}\text { Set common voltage of the Rx } \\
\text { for the } 40 \mathrm{MHz} \text { clock to } 1 / 2 \mathrm{vdd} \text {, } \\
\text { active high }\end{array}$ & 1'b1 \\
\hline 51 & enableTER_40M & REG_B_09[3] & $\begin{array}{l}\text { Enable internal termination of } \\
\text { the Rx for the } 40 \mathrm{MHz} \text { clock, } \\
\text { active high }\end{array}$ & 1'b1 \\
\hline 52 & invertData_40M & REG_B_09[2] & $\begin{array}{l}\text { Invert data of the Rx for the } 40 \\
\text { MHz clock, active high }\end{array}$ & 1'b0 \\
\hline 53 & equ_40M [1:0] & REG_B_09[1:0] & $\begin{array}{l}\text { Equalization strength of the Rx } \\
\text { for the } 40 \mathrm{MHz} \text { clock } \\
\text { 2'b00, equalization is turned off } \\
\text { 2'b11, largest equalization }\end{array}$ & 2'b00 \\
\hline 54 & enableRx_QInj & REG_B_OA[5] & $\begin{array}{l}\text { Enable the Rx for the QInj, } \\
\text { active high }\end{array}$ & 1'b1 \\
\hline
\end{tabular}


FERMILAB-TM-2746-CMS-E

\begin{tabular}{|c|c|c|c|c|}
\hline 55 & setCM_QInj & REG_B_OA[4] & $\begin{array}{l}\text { Set common voltage of the Rx } \\
\text { for the QInj to } 1 / 2 \text { vdd, active } \\
\text { high }\end{array}$ & 1'b1 \\
\hline 56 & enableTER_QInj & REG_B_OA[3] & $\begin{array}{l}\text { Enable internal termination of } \\
\text { the Rx for the QInj, active high }\end{array}$ & 1'b1 \\
\hline 57 & invertData_QInj & REG_B_OA[2] & $\begin{array}{l}\text { Invert data of the Rx for the } \\
\text { QInj, active high }\end{array}$ & 1'b0 \\
\hline 58 & equ_QInj [1:0] & REG_B_OA[1:0] & $\begin{array}{l}\text { Equalization strength of the Rx } \\
\text { for the QInj } \\
\text { 2'b00, equalization is turned off } \\
\text { 2'b11, largest equalization }\end{array}$ & 2'b00 \\
\hline 59 & AMPL_CLKTO[2:0] & REG_B_OB[2:0] & $\begin{array}{l}\text { 3bits to select different output } \\
\text { amplitude. } \\
3 b^{\prime} 000=\text { min amplitude }(50 \mathrm{~m}) \\
3 b^{\prime} 111=\text { max amplitude }(320 \mathrm{~m}) \\
\text { Step size } \approx 40 \mathrm{mV}\end{array}$ & 3’b111 \\
\hline 60 & disCML_CLKTO & REG_B_OB[3] & Disable CML driver, active high & 1'b0 \\
\hline 61 & AMPL_DOut[2:0] & REG_B_OB[6:4] & $\begin{array}{l}\text { 3bits to select different output } \\
\text { amplitude. } \\
3 b^{\prime} 000=\text { min amplitude }(50 \mathrm{~m}) \\
3 b^{\prime} 111=\text { max amplitude }(320 \mathrm{~m}) \\
\text { Step size } \approx 40 \mathrm{mV}\end{array}$ & 3’b111 \\
\hline & disCML_DOut & REG_B_OB[7] & Disable CML driver, active high & 1'b0 \\
\hline \multicolumn{5}{|l|}{62} \\
\hline 63 & & & & \\
\hline
\end{tabular}

a. See the pixel index map below 
FERMILAB-TM-2746-CMS-E

\begin{tabular}{|c|c|c|c|}
\hline $\begin{array}{c}P_{0} 0 \\
\text { index: } 0\end{array}$ & $\begin{array}{c}\text { P_0_1 } \\
\text { index: } 4\end{array}$ & $\begin{array}{c}\text { P_0_2 } \\
\text { index: } 8\end{array}$ & $\begin{array}{c}P_{0} 0_{3} \\
\text { index: } 12\end{array}$ \\
\hline $\begin{array}{c}P_{1}^{1} 0 \\
\text { index: } 1\end{array}$ & $\begin{array}{c}P_{1}^{1} 1 \\
\text { index: } 5\end{array}$ & $\begin{array}{c}\text { P_1_2 } \\
\text { index: } 9\end{array}$ & $\begin{array}{c}P_{1}^{1}-3 \\
\text { index: } 13\end{array}$ \\
\hline $\begin{array}{c}P_{2} 20 \\
\text { index: } 2\end{array}$ & $\begin{array}{c}\text { P_2_1 } \\
\text { index: } 6\end{array}$ & $\begin{array}{c}\text { P_2_2 } \\
\text { index: } 10\end{array}$ & $\begin{array}{c}\text { P_2_3 } \\
\text { index: } 14\end{array}$ \\
\hline $\begin{array}{c}P{ }_{3} 0 \\
\text { index: } 3\end{array}$ & $\begin{array}{c}P_{-3}{ }^{1} \\
\text { index: } 7\end{array}$ & $\begin{array}{c}P_{3}{ }^{2} \\
\text { index: } 11\end{array}$ & $\begin{array}{c}P_{3} 3 \\
\text { index: } 15\end{array}$ \\
\hline \multicolumn{4}{|c|}{ Chip peripherals } \\
\hline
\end{tabular}

Figure 4.1 Pixels index map 


\begin{tabular}{|c|c|c|c|c|c|c|c|c|c|}
\hline \multirow{2}{*}{$\begin{array}{l}\text { ADDR. } \\
\text { (Hex) }\end{array}$} & \multicolumn{8}{|l|}{ BIT } & \multirow{2}{*}{$\begin{array}{l}\text { Defaul } \\
\text { (Hex) }\end{array}$} \\
\hline & 7 & 6 & 5 & 4 & 3 & 2 & 1 & 0 & \\
\hline $\begin{array}{l}\text { REG_- } \\
\text { A_O0 }\end{array}$ & HysSel[3] & HysSel[2] & HysSel[1] & HysSel[0] & RfSel[1] & RfSel[0] & CLSel[1] & CLSel[0] & F8 \\
\hline $\begin{array}{l}\text { REG_- } \\
\text { A_01 }\end{array}$ & QSel[4] & QSel[3] & QSel[2] & QSel[1] & QSel[0] & IBSel[2] & IBSel[1] & IBSel[0] & 37 \\
\hline $\begin{array}{l}\text { REG } \\
\text { A_02 }\end{array}$ & $\begin{array}{l}\text { DIS_VTHInOu } \\
\text { t[7] }\end{array}$ & $\begin{array}{l}\text { DIS_VTHInO } \\
\text { ut [6] }\end{array}$ & $\begin{array}{l}\text { DIS_VTHIn } \\
\text { Out [5] }\end{array}$ & $\begin{array}{l}\text { DIS_VTHIn } \\
\text { Out [4] }\end{array}$ & $\begin{array}{l}\text { DIS_VTHIn } \\
\text { Out [3] }\end{array}$ & $\begin{array}{l}\text { DIS_VTHIn } \\
\text { Out [2] }\end{array}$ & $\begin{array}{l}\text { DIS_VTHIn } \\
\text { Out [1] }\end{array}$ & $\begin{array}{l}\text { DIS_VTHIn } \\
\text { Out [0] }\end{array}$ & $\mathrm{FF}$ \\
\hline $\begin{array}{l}\text { REG } \\
\text { A_03 }\end{array}$ & $\begin{array}{l}\text { DIS_VTHInOu } \\
\text { t[15] }\end{array}$ & $\begin{array}{l}\text { DIS_VTHInO } \\
\text { ut[14]p }\end{array}$ & $\begin{array}{l}\text { DIS_VTHIn } \\
\text { Out[13] }\end{array}$ & $\begin{array}{l}\text { DIS_VTHIn } \\
\text { Out[12] }\end{array}$ & $\begin{array}{l}\text { DIS_VTHIn } \\
\text { Out [11] }\end{array}$ & $\begin{array}{l}\text { DIS_VTHIn } \\
\text { Out [10] }\end{array}$ & $\begin{array}{l}\text { DIS_VTHIn } \\
\text { Out [9] }\end{array}$ & $\begin{array}{l}\text { DIS_VTHIn } \\
\text { Out [8] }\end{array}$ & $\mathrm{FF}$ \\
\hline REG & $\begin{array}{l}\text { EN_DiscriOut } \\
\text { [7] }\end{array}$ & $\begin{array}{l}\text { EN_Discrio } \\
\text { ut[6] }\end{array}$ & $\begin{array}{l}\text { EN_Discrio } \\
\text { ut[5] }\end{array}$ & $\begin{array}{l}\text { EN_Discrio } \\
\text { ut[4] }\end{array}$ & $\begin{array}{l}\text { EN_Discrio } \\
u t[3]\end{array}$ & $\begin{array}{l}\text { EN_Discrio } \\
\text { ut[2] }\end{array}$ & $\begin{array}{l}\text { EN_Discrio } \\
\text { ut[1] }\end{array}$ & $\begin{array}{l}\text { EN_Discrio } \\
\text { ut[0] }\end{array}$ & 11 \\
\hline $\begin{array}{l}\text { REG } \\
\text { A_05 }\end{array}$ & EN_QInj[7] & EN_QInj [6] & EN_QInj[5] & EN_QInj[4] & EN_QInj [3] & EN_QInj [2] & EN_QInj [1] & EN_QInj [0] & 01 \\
\hline $\begin{array}{l}\text { REG_ } \\
\text { A_06 }\end{array}$ & EN_QInj[15] & EN_QInj[14] & $\begin{array}{l}\text { EN_QInj[1 } \\
3]\end{array}$ & $\begin{array}{l}\text { EN_QInj[1 } \\
\text { 2] }\end{array}$ & $\begin{array}{l}\text { EN_QInj[11 } \\
]\end{array}$ & $\begin{array}{l}\text { EN_QInj[10 } \\
]\end{array}$ & EN_QInj [9] & EN_QInj [8] & 00 \\
\hline $\begin{array}{l}\text { REG } \\
\text { A } 07\end{array}$ & $x$ & RO_SEL & $\begin{array}{l}\text { DMRO_CO } \\
\text { L[1] }\end{array}$ & $\begin{array}{l}\text { DMRO_CO } \\
\text { L[0] }\end{array}$ & $\begin{array}{l}\text { OE_DMRO } \\
\text { Row[3] }\end{array}$ & $\begin{array}{l}\text { OE_DMRO } \\
\text { Row[2] }\end{array}$ & $\begin{array}{l}\text { OE_DMRO } \\
\text { Row[1] }\end{array}$ & $\begin{array}{l}\text { OE_DMRO } \\
\text { Row[0] }\end{array}$ & 01 \\
\hline $\begin{array}{l}\text { REG_ } \\
\text { A_08 }\end{array}$ & $\begin{array}{l}\text { PD_DACDiscri } \\
\text { [7] }\end{array}$ & $\begin{array}{l}\text { PD_DACDis } \\
\text { cri [6] }\end{array}$ & $\begin{array}{l}\text { PD_DACDis } \\
\text { cri [5] }\end{array}$ & $\begin{array}{l}\text { PD_DACDis } \\
\text { cri [4] }\end{array}$ & $\begin{array}{l}\text { PD_DACDis } \\
\text { cri [3] }\end{array}$ & $\begin{array}{l}\text { PD_DACDis } \\
\text { cri [2] }\end{array}$ & $\begin{array}{l}\text { PD_DACDis } \\
\text { cri [1] }\end{array}$ & $\begin{array}{l}\text { PD_DACDis } \\
\text { cri [0] }\end{array}$ & 00 \\
\hline $\begin{array}{l}\text { REG } \\
\text { A_09 }\end{array}$ & $\begin{array}{l}\text { PD_DACDiscri } \\
{[15]}\end{array}$ & $\begin{array}{l}\text { PD_DACDis } \\
\text { cri[14] }\end{array}$ & $\begin{array}{l}\text { PD_DACDis } \\
\text { cri[13] }\end{array}$ & $\begin{array}{l}\text { PD_DACDis } \\
\text { cri[12] }\end{array}$ & $\begin{array}{l}\text { PD_DACDis } \\
\text { cri [11] }\end{array}$ & $\begin{array}{l}\text { PD_DACDis } \\
\text { cri [10] }\end{array}$ & $\begin{array}{l}\text { PD_DACDis } \\
\text { cri [9] }\end{array}$ & $\begin{array}{l}\text { PD_DACDis } \\
\text { cri [8] }\end{array}$ & 00 \\
\hline $\begin{array}{l}\text { REG } \\
\text { A_OA }\end{array}$ & VTHIn[7] & VTHIn[6] & VTHIn[5] & VTHIn[4] & VTHIn[3] & VTHIn[2] & VTHIn[1] & VTHIn[0] & 00 \\
\hline $\begin{array}{l}\text { REG } \\
\mathrm{A} \_\mathrm{OB}\end{array}$ & VTHIn[15] & VTHIn[14] & VTHIn[13] & VTHIn[12] & VTHIn[11] & VTHIn[10] & VTHIn[9] & VTHIn[8] & 02 \\
\hline $\begin{array}{l}\text { REG } \\
\mathrm{A} O \mathrm{C}\end{array}$ & VTHIn[23] & VTHIn[22] & VTHIn[21] & VTHIn[20] & VTHIn[19] & VTHIn[18] & VTHIn[17] & VTHIn[16] & 08 \\
\hline $\begin{array}{l}\text { REG } \\
\text { A_OD }\end{array}$ & VTHIn[31] & VTHIn[30] & VTHIn[29] & VTHIn[28] & VTHIn[27] & VTHIn[26] & VTHIn[25] & VTHIn[24] & 20 \\
\hline $\begin{array}{l}\text { REG_- } \\
\text { A_OE }\end{array}$ & VTHIn[39] & VTHIn[38] & VTHIn[37] & VTHIn[36] & VTHIn[35] & VTHIn[34] & VTHIn[33] & VTHIn[32] & 80 \\
\hline $\begin{array}{l}\text { REG_- } \\
\text { A_OF }\end{array}$ & VTHIn[47] & VTHIn[46] & VTHIn[45] & VTHIn[44] & VTHIn[43] & VTHIn[42] & VTHIn[41] & VTHIn[40] & 00 \\
\hline $\begin{array}{l}\text { REG_ } \\
\text { A_10 }\end{array}$ & VTHIn[55] & VTHIn[54] & VTHIn[53] & VTHIn[52] & VTHIn[51] & VTHIn[50] & VTHIn[49] & VTHIn[48] & 02 \\
\hline $\begin{array}{l}\text { REG_ } \\
\text { A_11 }\end{array}$ & VTHIn[63] & VTHIn[62] & VTHIn[61] & VTHIn[60] & VTHIn[59] & VTHIn[58] & VTHIn[57] & VTHIn[56] & 08 \\
\hline $\begin{array}{l}\text { REG_ } \\
\text { A_12 }\end{array}$ & VTHIn[71] & VTHIn[70] & VTHIn[69] & VTHIn[68] & VTHIn[67] & VTHIn[66] & VTHIn[65] & VTHIn[64] & 20 \\
\hline $\begin{array}{l}\text { REG_- } \\
\text { A_13 }\end{array}$ & VTHIn[79] & VTHIn[78] & VTHIn[77] & VTHIn[76] & VTHIn[75] & VTHIn[74] & VTHIn[73] & VTHIn[72] & 80 \\
\hline $\begin{array}{l}\text { REG } \\
\text { A_14 }\end{array}$ & VTHIn[87] & VTHIn[86] & VTHIn[85] & VTHIn[84] & VTHIn[83] & VTHIn[82] & VTHIn[81] & VTHIn[80] & 00 \\
\hline REG_ & VTHIn[95] & VTHIn[94] & VTHIn[93] & VTHIn[92] & VTHIn[91] & VTHIn[90] & VTHIn[89] & VTHIn[88] & 02 \\
\hline $\begin{array}{l}\text { REG } \\
\text { A_16 }\end{array}$ & VTHIn[103] & VTHIn[102] & VTHIn[101] & VTHIn[100] & VTHIn[99] & VTHIn[98] & VTHIn[97] & VTHIn[96] & 08 \\
\hline $\begin{array}{l}\text { REG } \\
\text { A_17 }\end{array}$ & VTHIn[111] & VTHIn[110] & VTHIn[109] & VTHIn[108] & VTHIn[107] & VTHIn[106] & VTHIn[105] & VTHIn[104] & 20 \\
\hline $\begin{array}{l}\text { REG } \\
\text { A_18 }\end{array}$ & VTHIn[119] & VTHIn[118] & VTHIn[117] & VTHIn[116] & VTHIn[115] & VTHIn[114] & VTHIn[113] & VTHIn[112] & 80 \\
\hline $\begin{array}{l}\text { REG } \\
\text { A_19 }\end{array}$ & VTHIn[127] & VTHIn[126] & VTHIn[125] & VTHIn[124] & VTHIn[123] & VTHIn[122] & VTHIn[121] & VTHIn[120] & 00 \\
\hline REG & VTHIn[135] & VTHIn[134] & VTHIn[133] & VTHIn[132] & VTHIn[131] & VTHIn[130] & VTHIn[129] & VTHIn[128] & 02 \\
\hline $\begin{array}{l}\text { REG } \\
\text { A_1B }\end{array}$ & VTHIn[143] & VTHIn[142] & VTHIn[141] & VTHIn[140] & VTHIn[139] & VTHIn[138] & VTHIn[137] & VTHIn[136] & 08 \\
\hline $\begin{array}{l}\text { REG } \\
\text { A_1C }\end{array}$ & VTHIn[151] & VTHIn[150] & VTHIn[149] & VTHIn[148] & VTHIn[147] & VTHIn[146] & VTHIn[145] & VTHIn[144] & 20 \\
\hline
\end{tabular}


FERMILAB-TM-2746-CMS-E

\begin{tabular}{|c|c|c|c|c|c|c|c|c|c|}
\hline $\begin{array}{l}\text { REG } \\
\text { A_1D }\end{array}$ & VTHIn[159] & VTHIn[158] & VTHIn[157] & VTHIn[156] & VTHIn[155] & VTHIn[154] & VTHIn[153] & VTHIn[152] & 80 \\
\hline $\begin{array}{l}\text { REG } \\
\text { A_1E }\end{array}$ & ROI[7] & ROI[6] & ROI[5] & ROI[4] & $\mathrm{ROI}[3]$ & $\mathrm{ROI}[2]$ & ROI[1] & ROI[0] & $\mathrm{FF}$ \\
\hline $\begin{array}{l}\text { REG_ } \\
\text { A_1F }\end{array}$ & ROI[15] & ROI[14] & ROI[13] & ROI[12] & ROI[11] & ROI[10] & ROI[9] & $\mathrm{ROI}[8]$ & $\mathrm{FF}$ \\
\hline $\begin{array}{l}\text { REG_B } \\
\text { _00 }\end{array}$ & $\begin{array}{l}\text { timeStampM } \\
\text { ode_TDC }\end{array}$ & $\begin{array}{l}\text { testMode_T } \\
\text { DC }\end{array}$ & $\begin{array}{l}\text { selRawCod } \\
\text { E_TDC }\end{array}$ & $\begin{array}{l}\text { resetn_TD } \\
\text { C }\end{array}$ & $\begin{array}{l}\text { polaritySel } \\
\text { _TDC }\end{array}$ & $\begin{array}{l}\text { enable_TD } \\
\text { C }\end{array}$ & $\begin{array}{l}\text { enableMon } \\
\text { _TDC }\end{array}$ & $\begin{array}{l}\text { autoReset__ } \\
\text { TDC }\end{array}$ & $1 C$ \\
\hline $\begin{array}{l}\text { REG_B } \\
\text { 01 }\end{array}$ & $\mathrm{x}$ & $x$ & $x$ & $x$ & $\mathrm{x}$ & $\begin{array}{l}\text { level_TDC[ } \\
\text { 2] }\end{array}$ & $\begin{array}{l}\text { level_TDC[ } \\
\text { 1] }\end{array}$ & $\begin{array}{l}\text { level_TDC[ } \\
\text { 0] }\end{array}$ & 01 \\
\hline $\begin{array}{l}\text { REG_B } \\
\text { _02 }\end{array}$ & $x$ & $\begin{array}{l}\text { offset_TDC[ } \\
6]\end{array}$ & $\begin{array}{l}\text { offset_TDC } \\
{[5]}\end{array}$ & $\begin{array}{l}\text { offset_TDC } \\
{[4]}\end{array}$ & $\begin{array}{l}\text { offset_TDC[ } \\
3]\end{array}$ & $\begin{array}{l}\text { offset_TDC[ } \\
\text { 2] }\end{array}$ & $\begin{array}{l}\text { offset_TDC[ } \\
\text { 1] }\end{array}$ & $\begin{array}{l}\text { offset_TDC[ } \\
\text { 0] }\end{array}$ & 00 \\
\hline $\begin{array}{l}\text { REG_B } \\
\text { _03 }\end{array}$ & $x$ & $\begin{array}{l}\text { dllCPCurren } \\
t[3]\end{array}$ & $\begin{array}{l}\text { dllCPCurre } \\
\mathrm{nt}[2]\end{array}$ & $\begin{array}{l}\text { dllCPCurre } \\
\mathrm{nt}[1]\end{array}$ & $\begin{array}{l}\text { dllCPCurre } \\
\mathrm{nt}[0]\end{array}$ & dllCapReset & $\begin{array}{l}\text { dllForceDo } \\
\text { wn }\end{array}$ & dllEnable & 09 \\
\hline $\begin{array}{l}\text { REG_B } \\
\text { _04 }\end{array}$ & PhaseAdj[7] & PhaseAdj[6] & $\begin{array}{l}\text { PhaseAdj[5 } \\
\text { ] }\end{array}$ & $\begin{array}{l}\text { PhaseAdj[4 } \\
\text { ] }\end{array}$ & $\begin{array}{l}\text { PhaseAdj[3 } \\
\text { ] }\end{array}$ & $\begin{array}{l}\text { PhaseAdj[2 } \\
\text { ] }\end{array}$ & $\begin{array}{l}\text { PhaseAdj[1 } \\
\text { ] }\end{array}$ & $\begin{array}{l}\text { PhaseAdj }[0 \\
]\end{array}$ & 00 \\
\hline $\begin{array}{l}\text { REG_B } \\
\text { _05 }\end{array}$ & RefStrSel[7] & RefStrSel[6] & $\begin{array}{l}\text { RefStrSel[5 } \\
\text { ] }\end{array}$ & $\begin{array}{l}\text { RefStrSel[4 } \\
\text { ] }\end{array}$ & RefStrSel[3] & RefStrSel[2] & RefStrSel[1] & RefStrSel[0] & 03 \\
\hline $\begin{array}{l}\text { REG_B } \\
\text { 06 }\end{array}$ & $\mathrm{x}$ & CLKOutSel & TestCLK1 & TestCLKO & $\begin{array}{l}\text { TestMode_ } \\
\text { DMRO }\end{array}$ & $\begin{array}{l}\text { REVData_D } \\
\text { MRO }\end{array}$ & $\begin{array}{l}\text { REVCLK_D } \\
\text { MRO }\end{array}$ & $\begin{array}{l}\text { ENSCr_DM } \\
\text { RO }\end{array}$ & 41 \\
\hline $\begin{array}{l}\text { REG_B } \\
\text { _07 }\end{array}$ & $x$ & $x$ & $\begin{array}{l}\text { enableRx } \\
\text { 1P28G }\end{array}$ & $\begin{array}{l}\text { setCM_1P } \\
28 G\end{array}$ & $\begin{array}{l}\text { enableTER } \\
\text { 1P28G }\end{array}$ & $\begin{array}{l}\text { invertData } \\
\text { 1P28G }\end{array}$ & $\begin{array}{l}\text { equ_1P28G } \\
{[1]}\end{array}$ & $\begin{array}{l}\text { equ_1P28G } \\
{[0]}\end{array}$ & 38 \\
\hline $\begin{array}{l}\text { REG_B } \\
\text { _08 }\end{array}$ & $x$ & $x$ & $\begin{array}{l}\text { enableRx } \\
320 \mathrm{M}\end{array}$ & $\begin{array}{l}\text { setCM_32 } \\
\text { OM }\end{array}$ & $\begin{array}{l}\text { enableTER } \\
320 \mathrm{M}\end{array}$ & $\begin{array}{l}\text { invertData_ } \\
320 \mathrm{M}\end{array}$ & $\begin{array}{l}\text { equ_320M } \\
\text { [1] }\end{array}$ & $\begin{array}{l}\text { equ_320M } \\
{[0]}\end{array}$ & 18 \\
\hline $\begin{array}{l}\text { REG_B } \\
\text { _09 }\end{array}$ & $x$ & $x$ & $\begin{array}{l}\text { enableRx_ } \\
40 \mathrm{M}\end{array}$ & $\begin{array}{l}\text { setCM_40 } \\
\mathrm{M}\end{array}$ & $\begin{array}{l}\text { enableTER_ } \\
40 \mathrm{M}\end{array}$ & $\begin{array}{l}\text { invertData_ } \\
40 \mathrm{M}\end{array}$ & $\begin{array}{l}\text { equ_40M } \\
\text { [1] }\end{array}$ & $\begin{array}{l}\text { equ_40M } \\
{[0]}\end{array}$ & 18 \\
\hline $\begin{array}{l}\text { REG_B } \\
\text { _OA }\end{array}$ & $x$ & $x$ & $\begin{array}{l}\text { enableRx_ } \\
\text { QInj }\end{array}$ & $\begin{array}{l}\text { setCM_ } \\
\text { QInj }\end{array}$ & $\begin{array}{l}\text { enableTER_ } \\
\text { QInj }\end{array}$ & $\begin{array}{l}\text { invertData_ } \\
\text { QInj }\end{array}$ & $\begin{array}{l}\text { equ_QInj } \\
\text { [1] }\end{array}$ & $\begin{array}{l}\text { equ_QInj } \\
\text { [0] }\end{array}$ & 38 \\
\hline $\begin{array}{l}\text { REG_B } \\
\text { OB }\end{array}$ & disCML_DOut & $\begin{array}{l}\text { AMPL_DOu } \\
t[2]\end{array}$ & $\begin{array}{l}\text { AMPL_DO } \\
\text { ut[1] }\end{array}$ & $\begin{array}{l}\text { AMPL_DO } \\
\text { ut[0] }\end{array}$ & $\begin{array}{l}\text { disCML_CL } \\
\text { KTO }\end{array}$ & $\begin{array}{l}\text { AMPL_CLK } \\
\text { TO[2] }\end{array}$ & $\begin{array}{l}\text { AMPL_CLK } \\
\text { TO[1] }\end{array}$ & $\begin{array}{l}\text { AMPL_CLK } \\
\text { TO[0] }\end{array}$ & 77 \\
\hline
\end{tabular}

4.2 SLOW CONTROL FOR FULL PIXEL

A generic $12 \mathrm{C}$ slave is used in ETROC1 Full Pixel Test Block. The slave provides 32 bytes for reading and 16 bytes for writing by ETROC1. A 4-bit chip ID and a 4-bit chip reversion are available as well. The registers in the I2C slave are triplicated to mitigate SEU. The Slave addresses is 7'b1001110.

\begin{tabular}{|l|l|l|l|l|}
\hline Num. & REG. NAME & $\begin{array}{l}\text { REG. } \\
\text { ADDR. }\end{array}$ & Description & Default \\
\hline 1 & autoReset_TDC & REG_00[0] & TDC autoReset mode & 1'b0 \\
\hline 2 & enableMon_TDC & REG_00[1] & $\begin{array}{l}\text { Control of readout test mode in } \\
\text { ROTestGen. }\end{array}$ & $1^{\prime}$ b0 \\
\hline 3 & enable_TDC & REG_00[2] & Enable TDC & 1'b1 \\
\hline 4 & polaritySel_TDC & REG_00[3] & $\begin{array}{l}\text { TDC Controller control signal } \\
\text { polarity select }\end{array}$ & 1'b1 \\
\hline 5 & resetn_TDC & REG_00[4] & Reset TDC, low active & 1'b1 \\
\hline 6 & selRawCode_TDC & REG_00[5] & $\begin{array}{l}\text { Select Row data or combination } \\
\text { data }\end{array}$ & 1'b0 \\
\hline
\end{tabular}




\begin{tabular}{|c|c|c|c|c|}
\hline 7 & testMode_TDC & REG_00[6] & TDC test mode select & 1'b0 \\
\hline 8 & timeStampMode_TDC & REG_00[7] & $\begin{array}{l}\text { Calibration data timestamp } \\
\text { mode }\end{array}$ & 1'b0 \\
\hline 9 & level_TDC[2:0] & REG_01[2:0] & Bubble level & 3'b001 \\
\hline 10 & offset_TDC[6:0] & REG_02[6:0] & Ripple counter window offest & 7'b0000000 \\
\hline 11 & dllEnable & REG_03[0] & $\begin{array}{l}\text { Enable loop control of DLL. The } \\
\text { control voltage is tied to } \\
\text { ground when dllEnable==low. }\end{array}$ & 1'b1 \\
\hline 12 & dllForceDown & REG_03[1] & $\begin{array}{l}\text { Force to pull down the output } \\
\text { of the phase detector, active } \\
\text { high. }\end{array}$ & 1'b0 \\
\hline 13 & dllCapReset & REG_03[2] & $\begin{array}{l}\text { Reset the control voltage of DLL } \\
\text { to power supply, active high. }\end{array}$ & 1'b0 \\
\hline 14 & dlICPCurrent[3:0] & REG_03[6:3] & $\begin{array}{l}\text { Charge pump current control } \\
\text { bits, ranging from } 0 \text { to } 15 \mathrm{uA} \text { for } \\
\text { charge and discharge. }\end{array}$ & 4'b0001 \\
\hline 15 & dllLate & REG_20[0] & Lock status prompt. & $\begin{array}{l}\text { Read from } \\
\text { ETROC1 }\end{array}$ \\
\hline 16 & PhaseAdj[7:0] & REG_04 & $\begin{array}{l}\text { Phase selecting control bits, } \\
\text { PhaseAdj }<7: 3>\text { for coarse, } \\
\text { PhaseAdj }<2: 0>\text { for fine. }\end{array}$ & $8^{\prime} b 00000000$ \\
\hline 17 & RefStrSel[7:0] & REG_05 & TDC reference strobe selection. & 8'b00000011, \\
\hline 18 & RSTN_DMRO & REG_06[0] & DMRO Reset, active low & 1'b1 \\
\hline 19 & ENScr_DMRO & REG_06[1] & Enable scrambling, active high & 1'b1 \\
\hline 20 & REVCLK_DMRO & REG_06[2] & $\begin{array}{l}\text { Reversing the clock used for } \\
\text { input data latch, active-high. } \\
\text { When REVData=0 data is } \\
\text { latched at the rising edges of } \\
\text { CLKWord, otherwise data is } \\
\text { latched at the falling edges of } \\
\text { CLKWord. }\end{array}$ & 1'b0 \\
\hline 21 & REVData_DMRO & REG_06[3] & $\begin{array}{l}\text { reversing input data, active- } \\
\text { high. }\end{array}$ & 1'b0 \\
\hline 22 & TestMode_DMRO & REG_06[4] & $\begin{array}{l}\text { Test mode input, active high. } \\
\text { The PRBS7 is sent out in test } \\
\text { mode (TestMode }==1 \text { ) while } \\
\text { the data is sent out in normal } \\
\text { mode (TestMode }==1 \text { ). }\end{array}$ & 1'b0 \\
\hline 23 & TestCLKO & REG_06[5] & $\begin{array}{l}\text { When TestCLKO }=1 \text {, the phase } \\
\text { shifter is bypassed and off-chip } \\
40 \mathrm{MHz} \text { and } 320 \mathrm{MHz} \text { are used. }\end{array}$ & 1'b0 \\
\hline 24 & TestCLK1 & REG_06[6] & $\begin{array}{l}\text { When TestCLK } 1=1 \text {, the TDC } \\
\text { reference strobe generator is } \\
\text { bypassed and off-chip } 40 \mathrm{MHz} \\
\text { and } 320 \mathrm{MHz} \text { are used. }\end{array}$ & 1'b0 \\
\hline
\end{tabular}




\begin{tabular}{|c|c|c|c|c|}
\hline 25 & CLKOutSel & REG_06[7] & $\begin{array}{l}\text { Select output from either } 40 \\
\text { MHz clock or TDC reference } \\
\text { strobe } \\
\text { 1'b0: } 40 \mathrm{MHz} \text { clock } \\
\text { 1'b1: TDC reference strobe }\end{array}$ & 1'b1 \\
\hline 26 & enableRx_1P28G & REG_07[5] & $\begin{array}{l}\text { Enable the Rx for } 1.28 \mathrm{GHz} \\
\text { clock, active high }\end{array}$ & 1'b1 \\
\hline 27 & setCM_1P28G & REG_07[4] & $\begin{array}{l}\text { Set common voltage of the Rx } \\
\text { for the } 1.28 \mathrm{GHz} \text { clock to } 1 / 2 \text { vdd, } \\
\text { active high }\end{array}$ & 1'b1 \\
\hline 28 & enableTER_1P28G & REG_07[3] & $\begin{array}{l}\text { Enable internal termination of } \\
\text { the Rx for the } 1.28 \mathrm{GHz} \text { clock, } \\
\text { active high }\end{array}$ & 1'b1 \\
\hline 29 & invertData_1P28G & REG_07[2] & $\begin{array}{l}\text { Invert data of the Rx for the } \\
1.28 \mathrm{GHz} \text { clock, active high }\end{array}$ & $1^{\prime} \mathrm{b} 0$ \\
\hline 30 & equ_1P28G[1:0] & REG_07[1:0] & $\begin{array}{l}\text { Equalization strength of the Rx } \\
\text { for the } 320 \mathrm{MHz} \text { clock } \\
\text { 2'b00, equalization is turned off } \\
\text { 2'b11, largest equalization }\end{array}$ & $2^{\prime} \mathrm{b} 00$ \\
\hline 31 & enableRx_320M & REG_08[5] & $\begin{array}{l}\text { Enable the Rx for the } 320 \mathrm{MHz} \\
\text { clock, active high }\end{array}$ & 1'b0 \\
\hline 32 & setCM_320M & REG_08[4] & $\begin{array}{l}\text { Set common voltage of the Rx } \\
\text { for the } 320 \mathrm{MHz} \text { clock to } 1 / 2 \mathrm{vdd} \text {, } \\
\text { active high }\end{array}$ & 1'b1 \\
\hline 33 & enableTER_320M & REG_08[3] & $\begin{array}{l}\text { Enable internal termination of } \\
\text { the Rx for the } 320 \mathrm{MHz} \text { clock, } \\
\text { active high }\end{array}$ & 1'b1 \\
\hline 34 & invertData_320M & REG_08[2] & $\begin{array}{l}\text { Invert data of the Rx for the } \\
320 \mathrm{MHz} \text { clock, active high }\end{array}$ & $1^{\prime} \mathrm{b} 0$ \\
\hline 35 & equ_320M [1:0] & REG_08[1:0] & $\begin{array}{l}\text { Equalization strength of the Rx } \\
\text { for the } 320 \mathrm{MHz} \text { clock } \\
\text { 2'b00, equalization is turned off } \\
\text { 2'b11, largest equalization }\end{array}$ & 2'b00 \\
\hline 36 & enableRx_40M & REG_09[5] & $\begin{array}{l}\text { Enable the Rx for the } 40 \mathrm{MHz} \\
\text { clock, active high }\end{array}$ & $1^{\prime} \mathrm{b} 0$ \\
\hline 37 & setCM_40M & REG_09[4] & $\begin{array}{l}\text { Set common voltage of the Rx } \\
\text { for the } 40 \mathrm{MHz} \text { clock to } 1 / 2 \mathrm{vdd} \text {, } \\
\text { active high }\end{array}$ & 1'b1 \\
\hline 38 & enableTER_40M & REG_09[3] & $\begin{array}{l}\text { Enable internal termination of } \\
\text { the Rx for the } 40 \mathrm{MHz} \text { clock, } \\
\text { active high }\end{array}$ & 1'b1 \\
\hline 39 & invertData_40M & REG_09[2] & $\begin{array}{l}\text { Invert data of the Rx for the } 40 \\
\text { MHz clock, active high }\end{array}$ & $1^{\prime} \mathrm{b} 0$ \\
\hline 40 & equ_40M [1:0] & REG_09[1:0] & $\begin{array}{l}\text { Equalization strength of the Rx } \\
\text { for the } 40 \mathrm{MHz} \text { clock }\end{array}$ & $2^{\prime} \mathrm{b} 00$ \\
\hline
\end{tabular}


FERMILAB-TM-2746-CMS-E

\begin{tabular}{|c|c|c|c|c|}
\hline & & & $\begin{array}{l}2^{\prime} \text { b00, equalization is turned off } \\
2^{\prime} \text { b11, largest equalization }\end{array}$ & \\
\hline 41 & enableRx_QInj & REG_OA[5] & $\begin{array}{l}\text { Enable the Rx for the QInj, } \\
\text { active high }\end{array}$ & 1'b1 \\
\hline 42 & setCM_QInj & REG_OA[4] & $\begin{array}{l}\text { Set common voltage of the Rx } \\
\text { for the QInj to } 1 / 2 \text { vdd, active } \\
\text { high }\end{array}$ & 1'b1 \\
\hline 43 & enableTER_QInj & REG_OA[3] & $\begin{array}{l}\text { Enable internal termination of } \\
\text { the Rx for the QInj, active high }\end{array}$ & 1'b1 \\
\hline 44 & invertData_QInj & REG_OA[2] & $\begin{array}{l}\text { Invert data of the Rx for the } \\
\text { QInj, active high }\end{array}$ & 1'b0 \\
\hline 45 & equ_QInj [1:0] & REG_0A[1:0] & $\begin{array}{l}\text { Equalization strength of the Rx } \\
\text { for the QInj } \\
2^{\prime} \text { b00, equalization is turned off } \\
2^{\prime} \text { b11, largest equalization }\end{array}$ & 2'b00 \\
\hline 46 & AMPL_CLKTO[2:0] & REG_OB[2:0] & $\begin{array}{l}\text { 3bits to select different output } \\
\text { amplitude. } \\
3 b^{\prime} 000=\text { min amplitude }(50 \mathrm{~m}) \\
3 b^{\prime} 111=\text { max amplitude }(320 \mathrm{~m}) \\
\text { Step size } \approx 40 \mathrm{mV}\end{array}$ & 3'b111 \\
\hline 47 & disCML_CLKTO & REG_0B[3] & Disable CML driver, active high & 1'b0 \\
\hline 48 & AMPL_DOut[2:0] & REG_OB[6:4] & $\begin{array}{l}\text { 3bits to select different output } \\
\text { amplitude. } \\
3 b^{\prime} 000=\text { min amplitude }(50 \mathrm{~m}) \\
3 b^{\prime} 111=\text { max amplitude }(320 \mathrm{~m}) \\
\text { Step size } \approx 40 \mathrm{mV}\end{array}$ & 3'b111 \\
\hline 49 & disCML_DOut & REG_0B[7] & Disable CML driver, active high & 1'b0 \\
\hline 50 & CLSel[1:0] & REG_0C[1:0] & $\begin{array}{l}\text { Select of load capacitance of } \\
\text { the preamp first stage } \\
\text { 2'b00--> } 0 \mathrm{fC} \\
2^{`} \text { b01--> } 80 \mathrm{fC} \\
2^{`} \text { b10--> } 80 \mathrm{fC} \\
\text { 2'b01--> } 160 \mathrm{fC} \\
\text { Shared by all the pixels }\end{array}$ & 2'b00 \\
\hline 51 & RfSel[1:0] & REG_OC[3:2] & $\begin{array}{l}\text { Feedback resistance selection } \\
\text { 2`b00--> } 20 \mathrm{kOHm} \\
\text { 2`b01--> } 10 \mathrm{kOHm} \\
2^{`} \mathrm{~b} 10-->5.7 \mathrm{kOHm} \\
2^{`} \mathrm{~b} 11-->4.4 \mathrm{kOHm} \\
\text { Shared by all the pixels }\end{array}$ & 2`b10 \\
\hline 52 & HysSel[3:0] & REG_0C[7:4] & $\begin{array}{l}\text { Hysteresis voltage selection } \\
\text { 4`b0000 --> Vhys1 } \\
\text { 4`b0001 --> Vhys2 } \\
\text { 4`b0011 --> Vhys3 } \\
\text { 4`b0111 --> Vhys4 }\end{array}$ & 4`b1111 \\
\hline
\end{tabular}




\begin{tabular}{|c|c|c|c|c|}
\hline & & & $\begin{array}{l}\text { 4`b1111 --> Vhys5 } \\
\text { Vhys } 1>\text { Vhys } 2>\text { Vhys3 > Vhys } 4 \\
=\text { Vhys } 5=0 \\
\text { Shared by all the pixels }\end{array}$ & \\
\hline 53 & IBSel[2:0] & REG_0D[2:0] & $\begin{array}{l}\text { Bias current selection of the } \\
\text { input transistor in the preamp } \\
\text { 3`b000 --> I1 } \\
3^{`} b 001,3^{`} b 010,3^{`} b 100-->12 \\
3^{`} b 011,3^{`} b 110,3^{`} b 101-->13 \\
3^{`} b 111-->14 \\
\text { I1 > } 12>13>14 \\
\text { Shared by all the pixels }\end{array}$ & 3`b111 \\
\hline 54 & QSel[4:0] & REG_0D[7:3] & $\begin{array}{l}\text { Select injected charge, from } 1 \\
\mathrm{fC}\left(5^{`} \text { b00000) to } 32\right. \\
\mathrm{fC}\left(5^{`} \text { b11111) }\right. \\
\text { Typical charge from LGAD } \\
\text { sensor is } 7 \mathrm{fC}\left(7^{`} \text { b0000110) }\right. \\
\text { Shared by all the pixels }\end{array}$ & 5'b00110 \\
\hline 55 & VTHIn[9:0] & $\begin{array}{l}\text { REG_0E[7:0] } \\
\text { REG_OF[1:0] }\end{array}$ & $\begin{array}{l}\text { Threshold voltage input of } \\
\text { Discriminator, VTHIn[9:0] } \\
\text { represents DAC setting } \\
\text { REG_OF[1:0] go to VTHIn[9:8] }\end{array}$ & $10 ` b 1000000000$ \\
\hline 56 & EN_QInj & REG_OF[2] & enable the charge injection & 1'b1 \\
\hline 57 & EN_DiscriOut & REG_OF[3] & Enable Discriminator Output & 1'b0 \\
\hline 58 & Dis_VTHInOut & REG_OF[4] & Disable VTHIn Output & 1'b1 \\
\hline 59 & PD_DACDiscri & REG_OF[5] & $\begin{array}{l}\text { Power down the DAC and the } \\
\text { discriminator, active low }\end{array}$ & 1'b0 \\
\hline 60 & OE_DMRO & REG_OF[6] & Output enable of DMRO & 1'b1 \\
\hline
\end{tabular}

\subsection{SLOW CONTROL FOR TDC TEST BLOCK}

A generic I2C slave is used in ETROC1 TDC Test Block. The slave provides 32 bytes for writing and 16 bytes for reading by ETROC1. A 4-bit chip ID and a 4-bit chip reversion are available as well. The registers in the I2C slave are triplicated to mitigate SEU. The Slave addresses is 7'b010001X, The LSB of address is determined by pad $\mathrm{A} 0$.

\begin{tabular}{|l|l|l|l|l|}
\hline 1 & Dataout_disCMLDriver_BIAS & REG_00[0] & $\begin{array}{l}\text { Disable Dataout CML } \\
\text { Driver }\end{array}$ & 1'b0 \\
\hline 2 & Clk40Mout_disCMLDriver_BIAS & REG_00[1] & $\begin{array}{l}\text { Disable Clk40M CML } \\
\text { Driver }\end{array}$ & 1'b0 \\
\hline 3 & tdc_offset<6:0> & REG_01[6:0] & $\begin{array}{l}\text { TDC ripple counter } \\
\text { metastability window } \\
\text { offset. }\end{array}$ & 7'b0000000 \\
\hline
\end{tabular}




\begin{tabular}{|c|c|c|c|c|}
\hline 4 & tdc_enable & REG_01[7] & TDC enable & 1'b1 \\
\hline 5 & tdc_level<2:0> & REG_02[2:0] & $\begin{array}{l}\text { TDC Encoder bubble } \\
\text { tolerance. }\end{array}$ & 3'b001 \\
\hline 6 & tdc_testMode & REG_02[3] & TDC Test Mode & 1'b0 \\
\hline 7 & tdc_selRawCode & REG_02[4] & $\begin{array}{l}\text { Select TDC Raw code, } \\
\text { always "0" }\end{array}$ & 1'b0 \\
\hline 8 & tdc_resetn & REG_02[5] & TDC reset signal & 1'b1 \\
\hline 9 & tdc_polaritySel & REG_02[6] & $\begin{array}{l}\text { TDC controller output } \\
\text { signal polarity }\end{array}$ & 1'b1 \\
\hline 10 & tdc_autoReset & REG_02[7] & $\begin{array}{l}\text { TDC automatic reset } \\
\text { signal }\end{array}$ & 1'b0 \\
\hline 11 & Clk40Mout_AmplSel<2:0> & REG_03[2:0] & $\begin{array}{l}40 \mathrm{MHz} \text { clock CML } \\
\text { output Amplitude select }\end{array}$ & 3'b001 \\
\hline 12 & tdc_enableMon & REG_03[3] & $\begin{array}{l}\text { Enable TDC raw data } \\
\text { output }\end{array}$ & 1'b1 \\
\hline 13 & tdc_timeStampMode & REG_03[4] & $\begin{array}{l}\text { TDC Calibration data } \\
\text { timeStamp Mode }\end{array}$ & 1'b0 \\
\hline 14 & Dataout_AmplSel<2:0> & REG_04[2:0] & $\begin{array}{l}1.28 \mathrm{GHz} \text { Serial data } \\
\text { output Amplitude select }\end{array}$ & 3'b001 \\
\hline 15 & ro_testmode & REG_04[3] & DMRO test mode select & 1'b0 \\
\hline 16 & ro_enable & REG_04[4] & Enable DMRO & 1'b1 \\
\hline 17 & ro_reverse & REG_04[5] & $\begin{array}{l}\text { DMRO output data } \\
\text { reverse }\end{array}$ & 1'b0 \\
\hline 18 & ro_resetn & REG_04[6] & DMRO reset, low active & 1'b1 \\
\hline 19 & ro_revclk & REG_04[7] & $\begin{array}{l}\text { DMRO } 40 \mathrm{MHz} \text { clock } \\
\text { reverse }\end{array}$ & 1'b0 \\
\hline 20 & Dataout_Sel & REG_05[0] & $\begin{array}{l}\text { 1.28GHz data output } \\
\text { when asserted, } 320 \mathrm{MHz} \\
\text { clock pulse output when } \\
\text { deserted }\end{array}$ & 1'b1 \\
\hline 21 & Clk320M_Psel & REG_05[1] & $\begin{array}{l}\text { 320M Pulse clock comes } \\
\text { from external when } \\
\text { asserted, otherwise } \\
\text { comes from internal }\end{array}$ & 1'b1 \\
\hline 22 & Clk40M_Psel & REG_05[2] & $\begin{array}{l}\text { 40M Pulse clock comes } \\
\text { from external when } \\
\text { asserted, otherwise } \\
\text { comes from internal }\end{array}$ & 1'b1 \\
\hline 23 & Clk320M_Sel & REG_05[3] & $\begin{array}{l}\text { 320M clock comes from } \\
\text { internal divider when } \\
\text { asserted, otherwise }\end{array}$ & 1'b1 \\
\hline
\end{tabular}




\begin{tabular}{|c|c|c|c|c|}
\hline & & & $\begin{array}{l}\text { comes from external } \\
\text { input. }\end{array}$ & \\
\hline 24 & Clk40M_Sel & REG_05[4] & $\begin{array}{l}40 \mathrm{M} \text { clock comes from } \\
\text { internal divider when } \\
\text { asserted, otherwise } \\
\text { comes from external } \\
\text { input. }\end{array}$ & 1'b1 \\
\hline 25 & Pulse_Sel<7:0> & REG_06[7:0] & $\begin{array}{l}320 \mathrm{M} \text { clock pulse } \\
\text { location select }\end{array}$ & 8'b00000011 \\
\hline 26 & Clk40M_equalizer<1:0> & REG_07[1:0] & $\begin{array}{l}\text { 40M clock input eRx } \\
\text { equalizer intensity }\end{array}$ & 2'b00 \\
\hline 27 & Clk40M_invertData & REG_07[2] & $\begin{array}{l}40 \mathrm{M} \text { clock input eRx data } \\
\text { invert }\end{array}$ & 1'b0 \\
\hline 28 & Clk40M_enableTermination & REG_07[3] & $\begin{array}{l}\text { Enable } 40 \mathrm{M} \text { clock input } \\
\text { eRx termination }\end{array}$ & 1'b1 \\
\hline 29 & Clk40M_setCommonMode & REG_07[4] & $\begin{array}{l}\text { Set } 40 \mathrm{M} \text { clock input eRx } \\
\text { common mode }\end{array}$ & 1'b1 \\
\hline 30 & Clk40M_enableRx & REG_07[5] & $\begin{array}{l}\text { Enable 40M clock input } \\
\text { eRx }\end{array}$ & 1'b1 \\
\hline 31 & Clk320M_equalizer<1:0> & REG_08[1:0] & $\begin{array}{l}\text { 320M clock input eRx } \\
\text { equalizer intensity }\end{array}$ & 2'b00 \\
\hline 32 & Clk320M_invertData & REG_08[2] & $\begin{array}{l}\text { 320M clock input eRx } \\
\text { data invert }\end{array}$ & 1'b0 \\
\hline 33 & Clk320M_enableTermination & REG_08[3] & $\begin{array}{l}\text { Enable } 320 \mathrm{M} \text { clock input } \\
\text { eRx termination }\end{array}$ & 1'b1 \\
\hline 34 & Clk320M_setCommonMode & REG_08[4] & $\begin{array}{l}\text { Set } 320 \mathrm{M} \text { clock input eRx } \\
\text { common mode }\end{array}$ & 1'b1 \\
\hline 35 & Clk320M_enableRx & REG_08[5] & $\begin{array}{l}\text { Enable } 320 \mathrm{M} \text { clock input } \\
\text { eRx }\end{array}$ & 1'b1 \\
\hline 36 & Clk1G28_equalizer $<1: 0>$ & REG_09[1:0] & $\begin{array}{l}1.28 \mathrm{G} \text { clock input eRx } \\
\text { equalizer intensity }\end{array}$ & 2'b00 \\
\hline 37 & Clk 1G28_invertData & REG_09[2] & $\begin{array}{l}1.28 \mathrm{G} \text { clock input eRx } \\
\text { data invert }\end{array}$ & 1'b0 \\
\hline 38 & Clk1G28_enableTermination & REG_09[3] & $\begin{array}{l}\text { Enable } 1.28 \mathrm{G} \text { clock input } \\
\text { eRx termination }\end{array}$ & 1'b1 \\
\hline 39 & Clk1G28_setCommonMode & REG_09[4] & $\begin{array}{l}\text { Set } 1.28 \mathrm{G} \text { clock input eRx } \\
\text { common mode }\end{array}$ & 1'b1 \\
\hline 40 & Clk 1G28_enableRx & REG_09[5] & $\begin{array}{l}\text { Enable } 1.28 \mathrm{G} \text { clock input } \\
\text { eRx }\end{array}$ & 1'b1 \\
\hline 41 & Pulse_equalizer $<1: 0>$ & REG_0A[1:0] & $\begin{array}{l}\text { TDC pulse input eRx } \\
\text { equalizer intensity }\end{array}$ & 2'b00 \\
\hline
\end{tabular}


FERMILAB-TM-2746-CMS-E

\begin{tabular}{|l|l|l|l|l|}
\hline 42 & Pulse_invertData & REG_0A[2] & $\begin{array}{l}\text { TDC pulse input eRx data } \\
\text { invert }\end{array}$ & 1'b0 \\
\hline 43 & Pulse_enableTermination & REG_0A[3] & $\begin{array}{l}\text { Enable TDC pulse input } \\
\text { eRx termination }\end{array}$ & 1'b1 \\
\hline 44 & Pulse_setCommonMode & REG_0A[4] & $\begin{array}{l}\text { Set pulse input eRx } \\
\text { common mode }\end{array}$ & 1'b1 \\
\hline 45 & Pulse_enableRx & REG_0A[5] & Enable pulse input eRx & 1'b1 \\
\hline 46 & TDCRawData_Sel & REG_0B[0] & $\begin{array}{l}\text { TDC Raw data group } \\
\text { select }\end{array}$ & 1'b1 \\
\hline 47 & GRO_TOT_CK & REG_0B[1] & GRO TOT clock & 1'b1 \\
\hline 48 & GRO_TOTRST_N & REG_0B[2] & $\begin{array}{l}\text { GRO TOT Reset, active } \\
\text { low }\end{array}$ & 1'b1 \\
\hline 49 & GRO_TOA_Latch & REG_0B[3] & GRO TOA Latch clock & 1'b1 \\
\hline 50 & GRO_TOA_CK & REG_0B[4] & GRO TOA clock & 1'b1 \\
\hline 51 & GRO_TOARST_N & REG_0B[5] & $\begin{array}{l}\text { GRO TOA Reset, low } \\
\text { active }\end{array}$ & 1'b1 \\
\hline 52 & GRO_Start & REG_0B[6] & $\begin{array}{l}\text { GRO Start signal, high } \\
\text { active }\end{array}$ & 1'b0 \\
\hline 53 & GROout_disCMLDriver_BIASA/B/C & REG_0C[0] & $\begin{array}{l}\text { Disable GRO output CML } \\
\text { Driver }\end{array}$ & 1'b0 \\
\hline 54 & GROout_AmpISel<2:0> & REG_0C[3:1] & $\begin{array}{l}\text { GRO output CML Driver } \\
\text { Amplitude selection }\end{array}$ & 3'b001 \\
\hline
\end{tabular}

TDC Test block read-only register:

\begin{tabular}{|c|c|c|c|}
\hline & & TDCRawData_Sel $=0$ & TDCRawData_Sel = 1 \\
\hline 1 & REG_20[2:0] & CalCounterBMon<2:0> & TOACounterBMon<2:0> \\
\hline 2 & REG_20[5:3] & CalCounterAMon<2:0> & TOACounterAMon<2:0> \\
\hline 3 & REG_20[6] & CalerrorFlagReg & TOAerrorFalgReg \\
\hline 4 & REG_21[7:0] & TOT_codeReg<7:0> & TOARawDataMon<7:0> \\
\hline 5 & REG_22[7:0] & TOA_codeReg $\langle 6: 0\rangle$, TOT_codeReg $\langle 8\rangle$ & TOARawDataMon<15:8> \\
\hline 6 & REG_23[7:0] & Cal_codeReg<4:0>,TOA_codeReg<9:7> & TOARawDataMon<23:16> \\
\hline 7 & REG_24[7:0] & CalRawDataMon<31:29>,Cal_codeReg<9:5> & TOARawDataMon<31:24> \\
\hline 8 & REG_25[7:0] & CalRawDataMon<39:32> & TOARawDataMon<39:32> \\
\hline 9 & REG_26[7:0] & CalRawDataMon<47:40> & TOARawDataMon<47:40> \\
\hline 10 & REG_27[7:0] & CalRawDataMon<55:48> & TOARawDataMon<55:48> \\
\hline 11 & REG_28[6:0] & CalRawDataMon<62:56> & TOARawDataMon<62:56> \\
\hline 12 & REG_29[7:0] & $\begin{array}{l}\text { HitFalg, TOTerrorFlagReg, } \\
\text { TOTCounterAMon<2:0>, } \\
\text { TOTCounterBMon<2:0> }\end{array}$ & \\
\hline 13 & REG_2A[7:0] & CalRawDataMon<7:0> & TOTRawDataMon< $>$ :0> \\
\hline
\end{tabular}




\begin{tabular}{|l|l|l|l|}
\hline 14 & REG_2B[7:0] & CalRawDataMon $<15: 8>$ & TOTRawDataMon $<15: 8>$ \\
\hline 15 & REG_2C[7:0] & CalRawDataMon<23:16> & TOTRawDataMon $<23: 16>$ \\
\hline 16 & REG_2D[7:0] & $<* 3>$ Low,CalRawDataMon $<28: 24>$ & TOTRawDataMon $<31: 24>$ \\
\hline 17 & REG_2E[5:0] & DBF_QC $<5: 0>$ & RO_DBF_QC $<5: 0>$ \\
\hline
\end{tabular}

\section{BUILDING BLOCKS DESIGN}

\subsection{PREAMP}

The preamplifier consists of a two-stage amplifier. A cascode amplifier with resistive feedback acts as the first stage, and a source follower as the second stage. The size of each transistor in the preamplifier has been optimized by using an LGAD simulation as the input signal. The design considers both leading and trailing edge to optimize the TOT measurement for the time-walk correction. The feedback resistance is programmable to allow adjustment of the fall time, while the bias current is also programmable to allow different trade-offs between power consumption and performance. The load capacitance of the first stage is also programmable to allow optimization of the bandwidth.

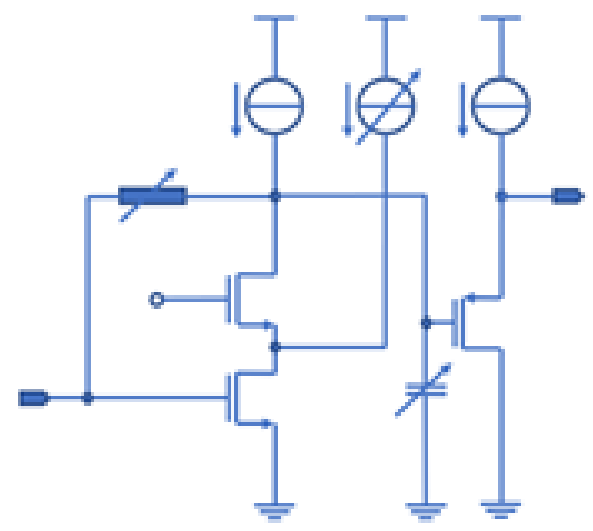

Figure 5.1 Block diagram of Preamplifier

\subsection{DISCRIMINATOR}

The discriminator, with a user programmable threshold controlled by an internal DAC, is optimized for the full chain for low signal size while keeping power consumption low. 


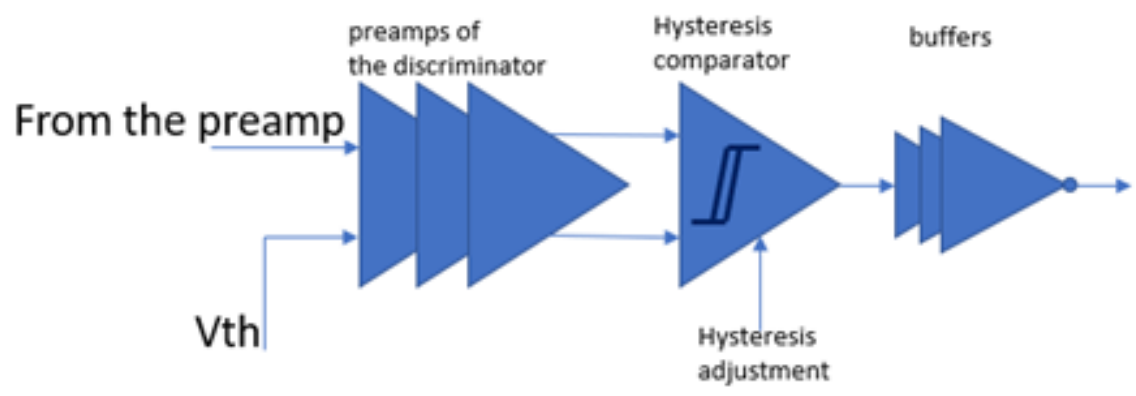

Figure 5.2 Block diagram of Discriminator

\subsection{TDC}

\subsubsection{TDC Core}

\subsubsection{Phase shifter}

The phase shifter circuit aims at producing a programmable phase rotation (up to 360 degree) with a time resolution of $97.6 \mathrm{ps}$ for $320 \mathrm{MHz}$ and $40 \mathrm{MHz}$ clock frequencies. The circuit is implemented as two functional blocks: a coarse phase shifter, with a fully digital implementation, and fine phase shifter, based on a Delay-Line Loop (DLL).

The coarse phase-shifter block is responsible for selecting the appropriate clock frequency and to set the clock phase with a resolution of 781.25 ps (period of $1.28 \mathrm{GHz}$ ), while the fine phase shifter further interpolates within the 781.25 ps interval down to 97.6 ps.

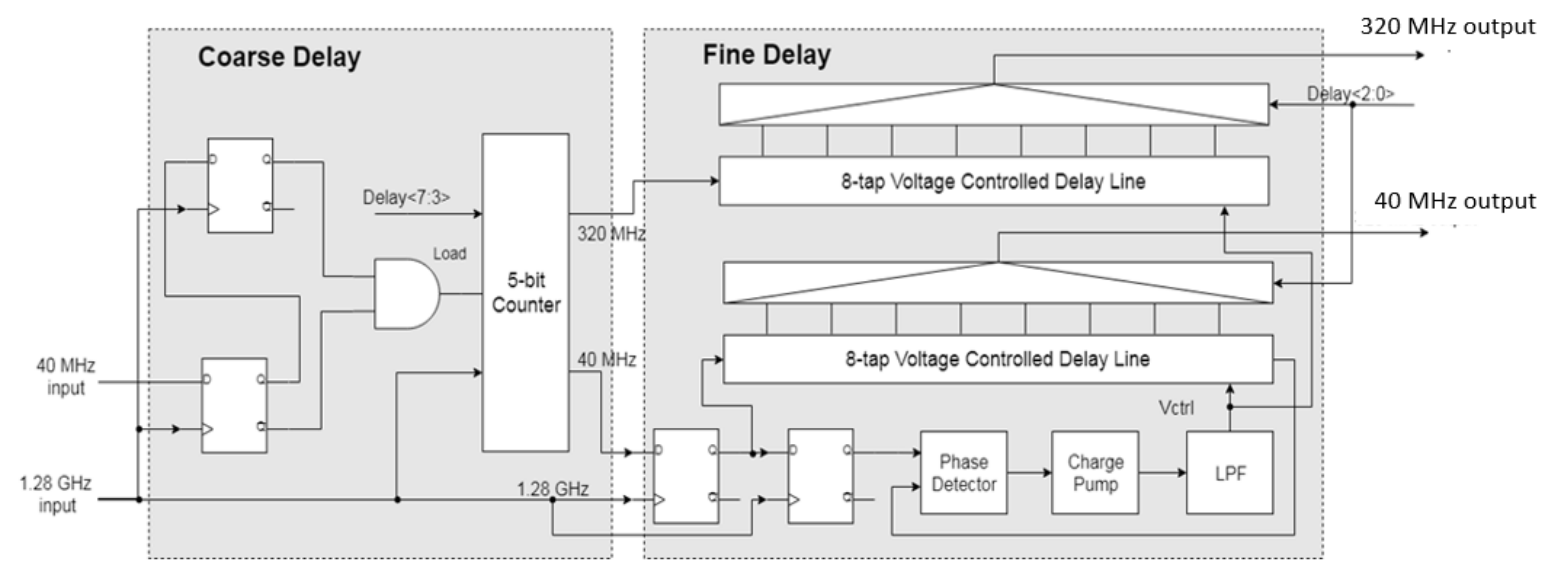

Figure 5.3.2-1 Block diagram of phase shifter

Table 5.3.2-1 IO interface definition of phase shifter

\begin{tabular}{|l|l|l|l|l|}
\hline NO & Name & Type & Description & Default \\
\hline
\end{tabular}




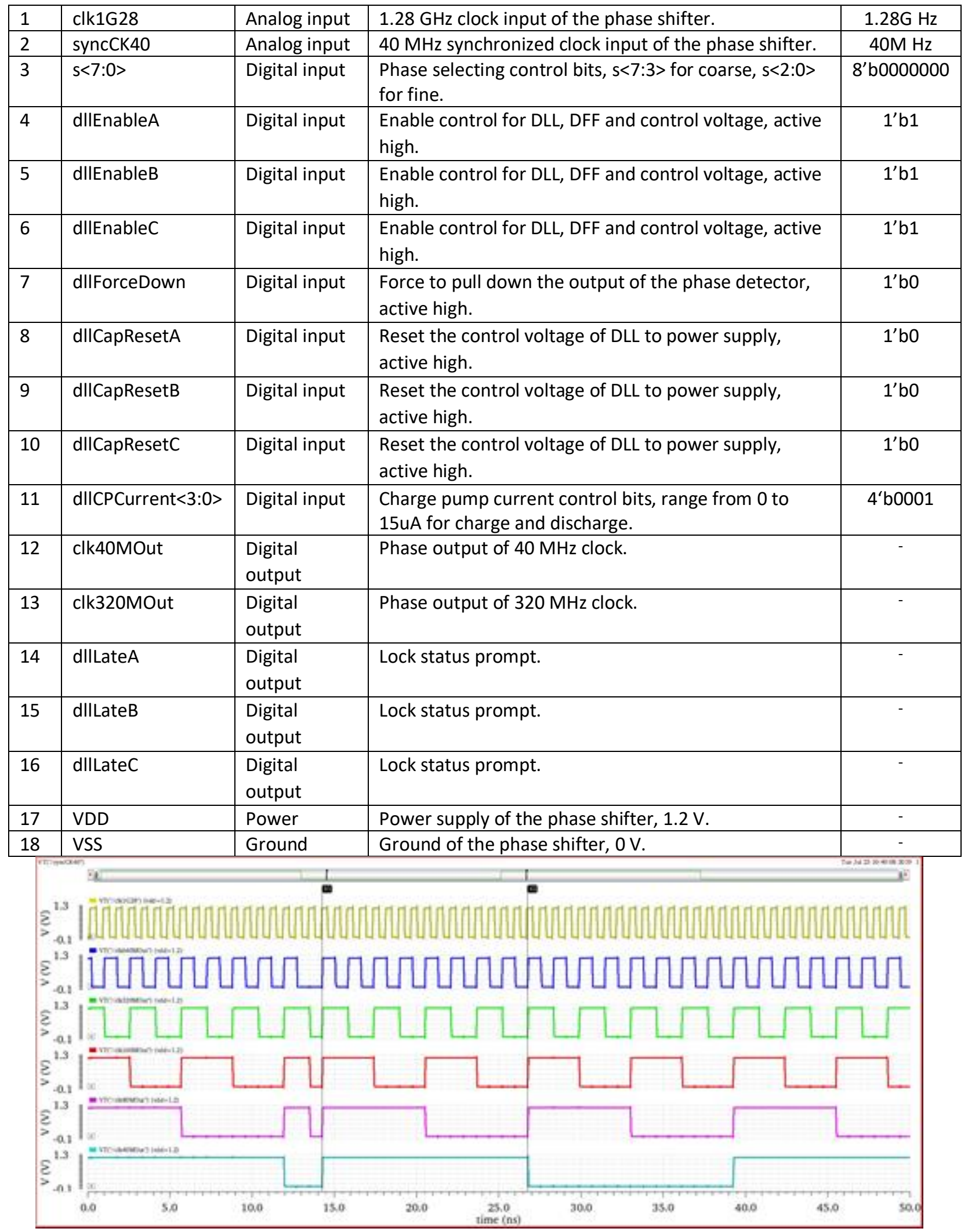


Figure 5.3.2-2 Post-layout simulation of coarse, Rising edge alignment

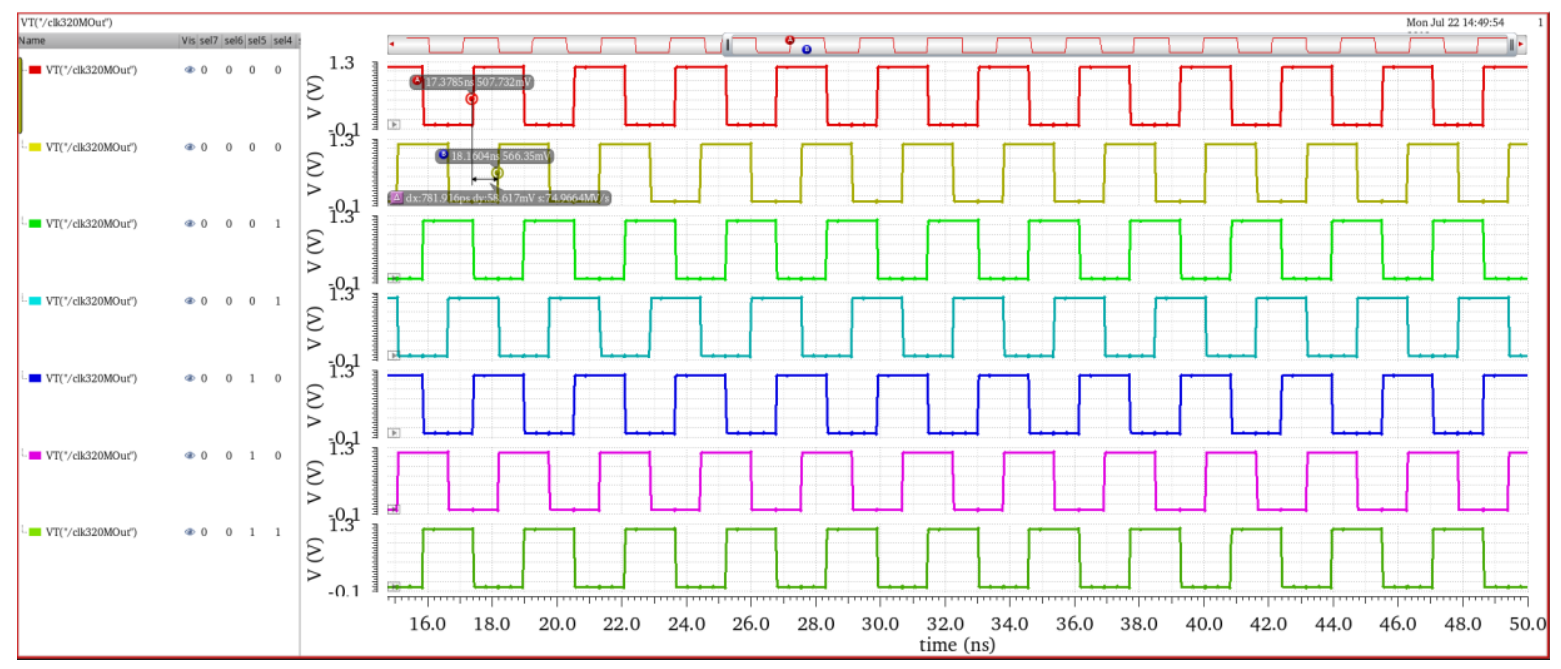

Figure 5.3.2-3 Simulation of coarse phase shifter,781.25 ps phase shift by coarse

\section{- Reset of the phase shifter}

a. After power on, the control voltage should be reset LPF to VDD by setting the "dllCapResetA/B/C" to a high level. The reset time is less than $200 \mathrm{~ns}$.

b. After resetting, the control voltage will continue to drop until the DLL is locked. Then it will change according to the switching of up and down signals in the charge pump.

\subsubsection{TDC reference strobe generator}

TDC reference strobe generator is a block designed for arbitrary-phase pulse generating. A $40 \mathrm{MHz}$ clock is the reference clock. The rising edge of the $40 \mathrm{MHz}$ clock is defined as the phase0. The synchronized $320 \mathrm{MHz}$ clock and the delays of $40 \mathrm{MHz}$ clock by DFF are used to generate the desired pulse. 8-bit control is adopted to select the output. 


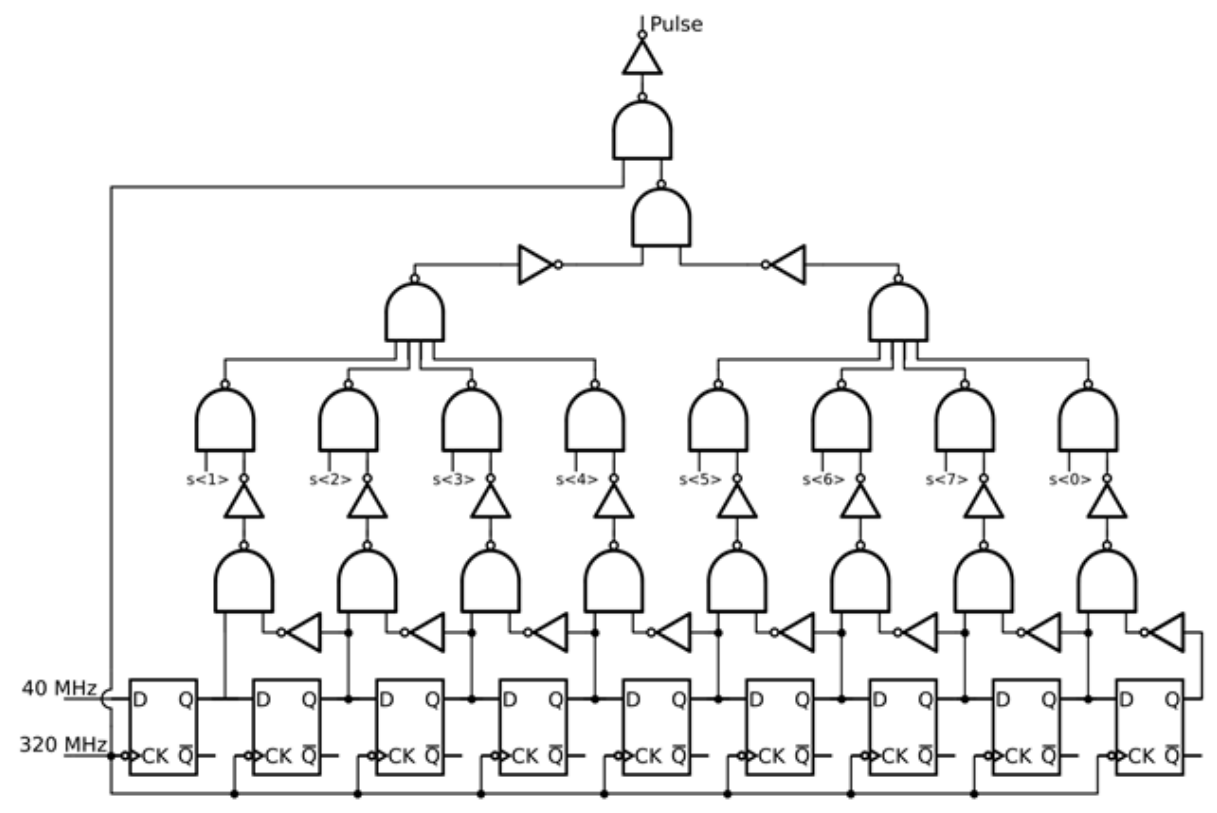

Figure 5.3.3-1 Block diagram of TDC pulse generator

Table 5.3.3-1 IO interface definition of TDC pulse generator

\begin{tabular}{|c|l|l|l|l|}
\hline \multicolumn{1}{|l|}{ NO } & Pin Name & Type & Description & Default \\
\hline 1 & CLK40M & Input & $40 \mathrm{MHz}$ reference clock. & \\
\hline 2 & CLK320M & Input & $320 \mathrm{MHz}$ reference clock. & \\
\hline 3 & s<7:0> & Input & $320 \mathrm{MHz}$ pulse phase select. & 8'b00000011 \\
\hline 4 & clk40_delay & Output & Delayed synchronized $40 \mathrm{MHz}$ clock. & \\
\hline 5 & clk320_pulse & Output & Pulse output controlled by s<7:0>. & \\
\hline 6 & VDD & Power & Power supply of TDC pulse generator, 1.2 V. & \\
\hline 7 & VSS & Power & Ground of TDC pulse generator, 0 V. & \\
\hline
\end{tabular}


FERMILAB-TM-2746-CMS-E

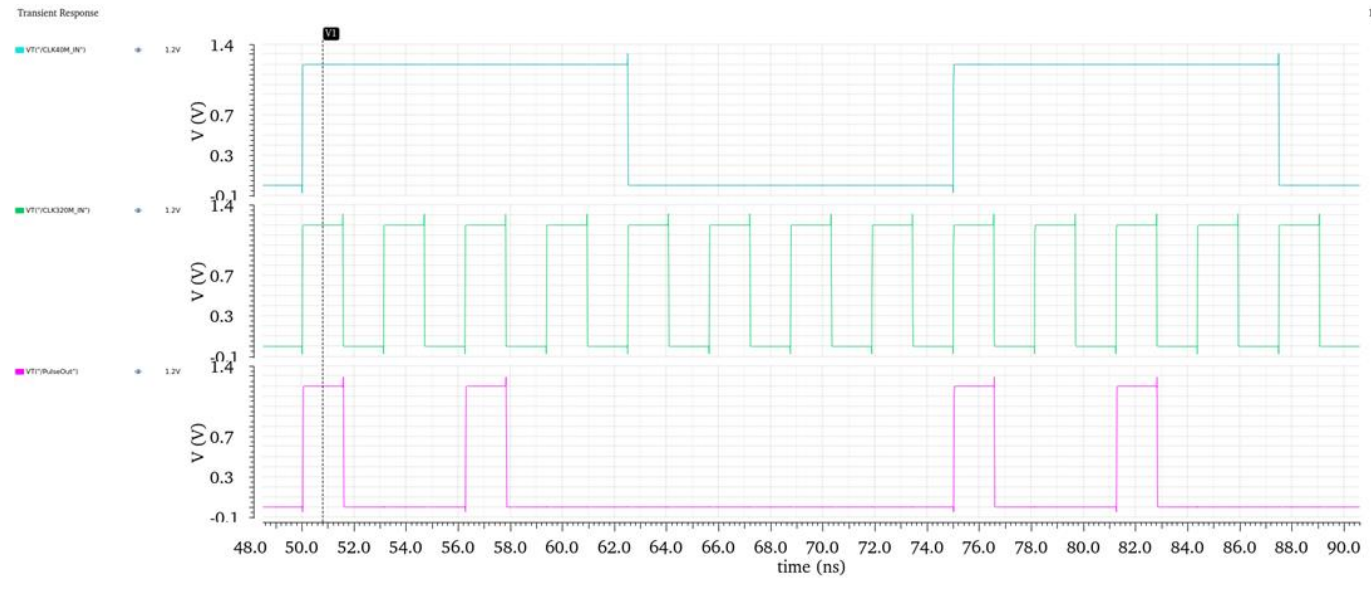

Figure 5.3.3-2 Post-layout simulation of TDC pulse generator, $s<7: 0>=2^{\prime} b 00000101$

\subsection{DMRO}

DMRO block takes 30-bit data as the input at the rate of $40 \mathrm{MHz}$, scrambles the input data with the polynomial of $X^{58}+X^{39}+1$, adds two bits as the header of the word( $\left.2^{\prime} b 10\right)$, and finally serializes 32-bit word to a $1.28 \mathrm{Gbps}$ data stream. A PRBS7 generator is integrated for testability consideration. MSB goes first when delivering a word serially. 


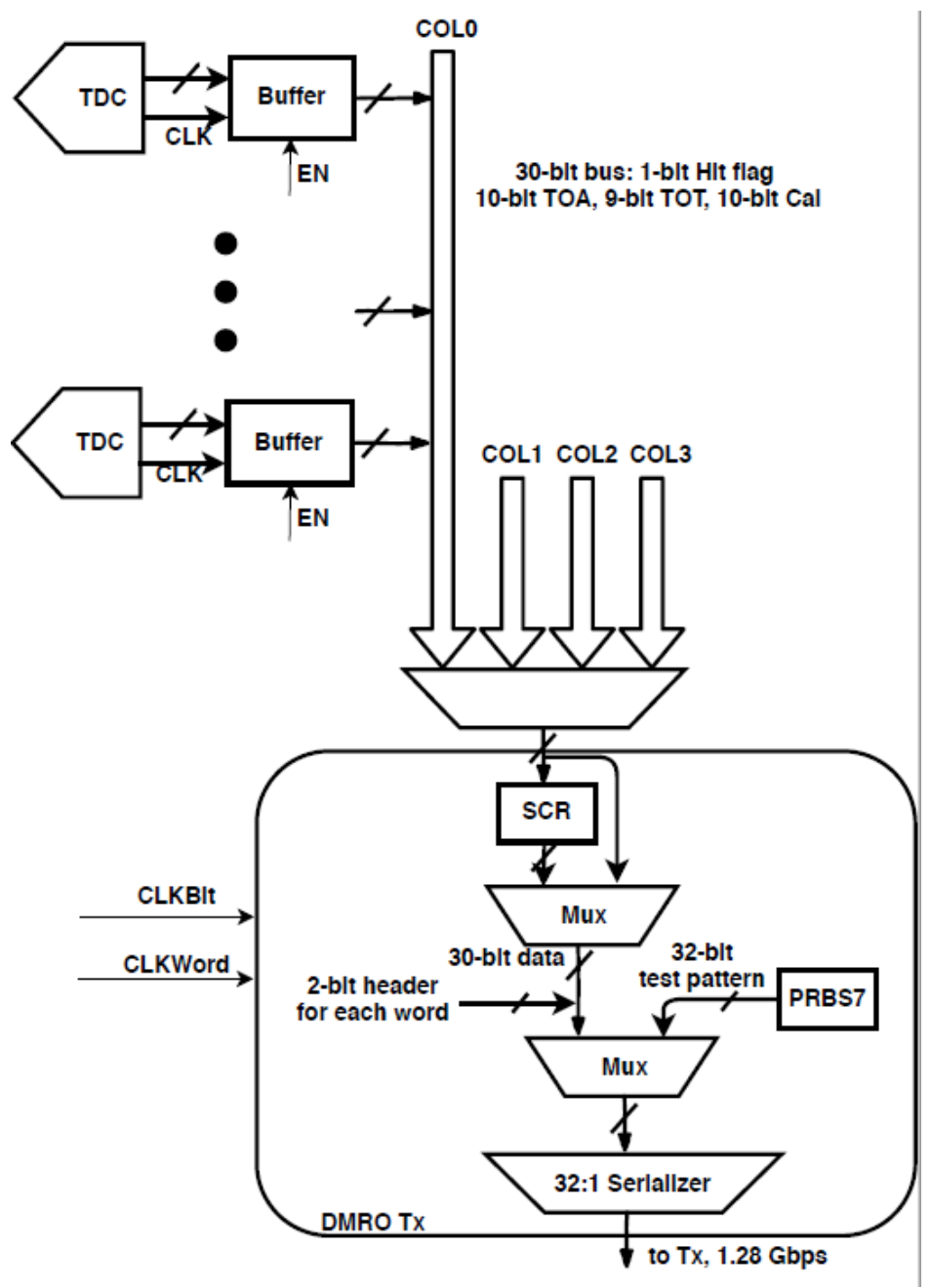

Figure 5.4.1 block diagram of the diagnostic mode readout scheme

Table 4.4 DMRO block pinout

\begin{tabular}{|l|l|l|l|}
\hline Num. & Pin name & Type & Description \\
\hline 1 & CLKBit & Digital input & $1.28 \mathrm{GHz}$ bit clock \\
\hline 2 & CLKWord & Digital input & $\begin{array}{l}40 \mathrm{MHz} \text { word clock. Input data is latched at falling } \\
\text { edges of this clock }\end{array}$ \\
\hline 3 & RSTn & Digital input & $\begin{array}{l}\text { Reset signal, active low. } \\
\text { The logic is reset asynchronously at falling edge of } \\
\text { RSTn and released synchronously at the first rising } \\
\text { edge of CLKWord after RSTn becoming high. }\end{array}$ \\
\hline 5 & REVData & Digital input & \begin{tabular}{l} 
reversing input data, active-high. \\
\hline
\end{tabular} \\
\hline REVCLK & Digital input & $\begin{array}{l}\text { Reversing the clock used for input data latch, active- } \\
\text { high. When REVData=0 data is latched at the rising } \\
\text { edges of CLKWord, otherwise data is latched at the } \\
\text { falling edges of CLKWord. }\end{array}$ \\
\hline
\end{tabular}




\begin{tabular}{|l|l|l|l|}
\hline & & & $\begin{array}{l}\text { Each time REVCLK changed, a reset pulse should be } \\
\text { applied to RSTn. }\end{array}$ \\
\hline 6 & ENScr & Digital input & Enable scrambling \\
\hline 7 & TestMode & Digital input & $\begin{array}{l}\text { Test mode input, active high. The PRBS7 is sent out in } \\
\text { test mode (TestMode == 1) while the data is sent out } \\
\text { in normal mode (TestMode == 0). }\end{array}$ \\
\hline 8 & Dataln[29:0] & Digital input & 30-bit data input \\
\hline 9 & DataOut & Digital output & Serialized word. \\
\hline
\end{tabular}

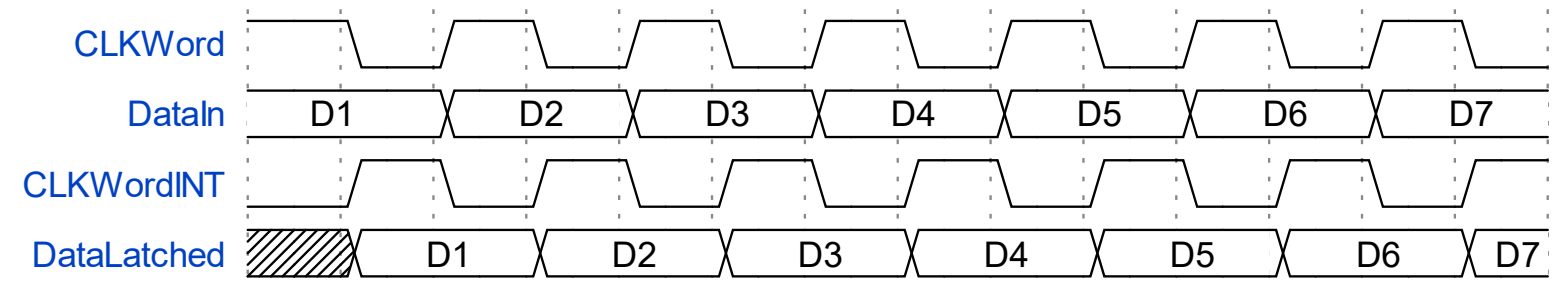

Figure 5.4.2 timing diagram of the DMRO input interface, REVCLK=1'b1

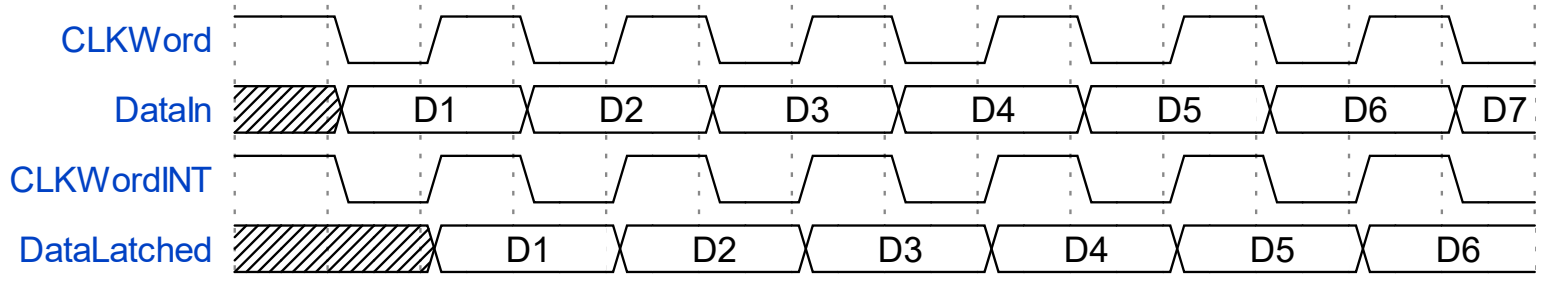

Figure 5.4.3 timing diagram of the DMRO input interface, REVCLK=1'bo

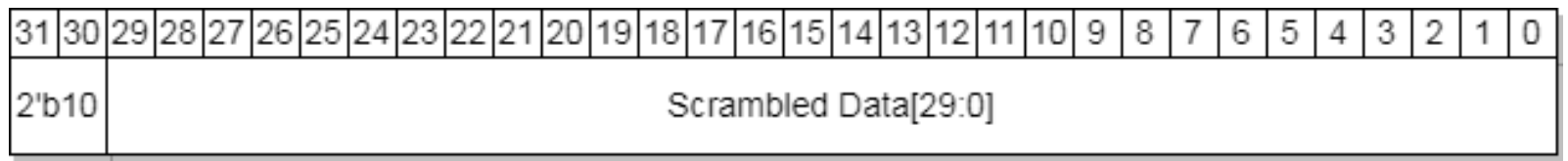

Figure 5.4.4 data format of the DMRO data when the scrambler is on

\section{$5.5 \mathrm{Tx}$}

Tx block takes the 1.28Gbps data whose amplitude is from 0 to vdd as input signal, translates the singleend signal into the signals in differential, finally drive $100 \mathrm{Ohm}$ impedance. Its output amplitude is programmable by 3 bits control as well.

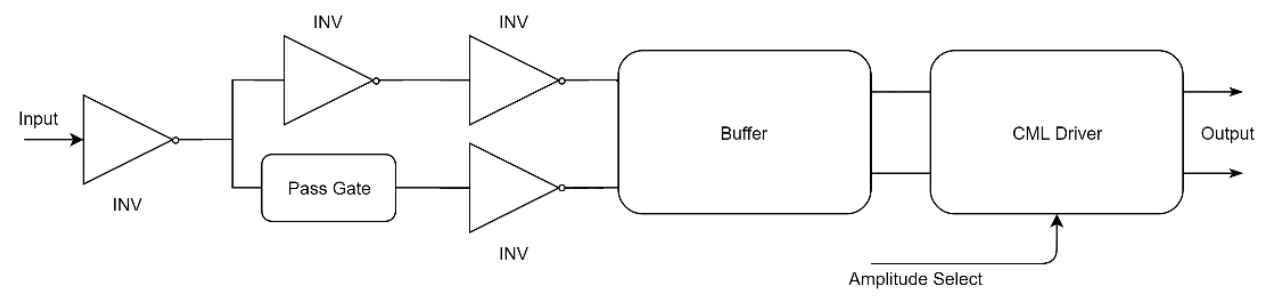


Figure 5.5.1 Diagram of $T x$

Table 5.5.1 Tx block pinout

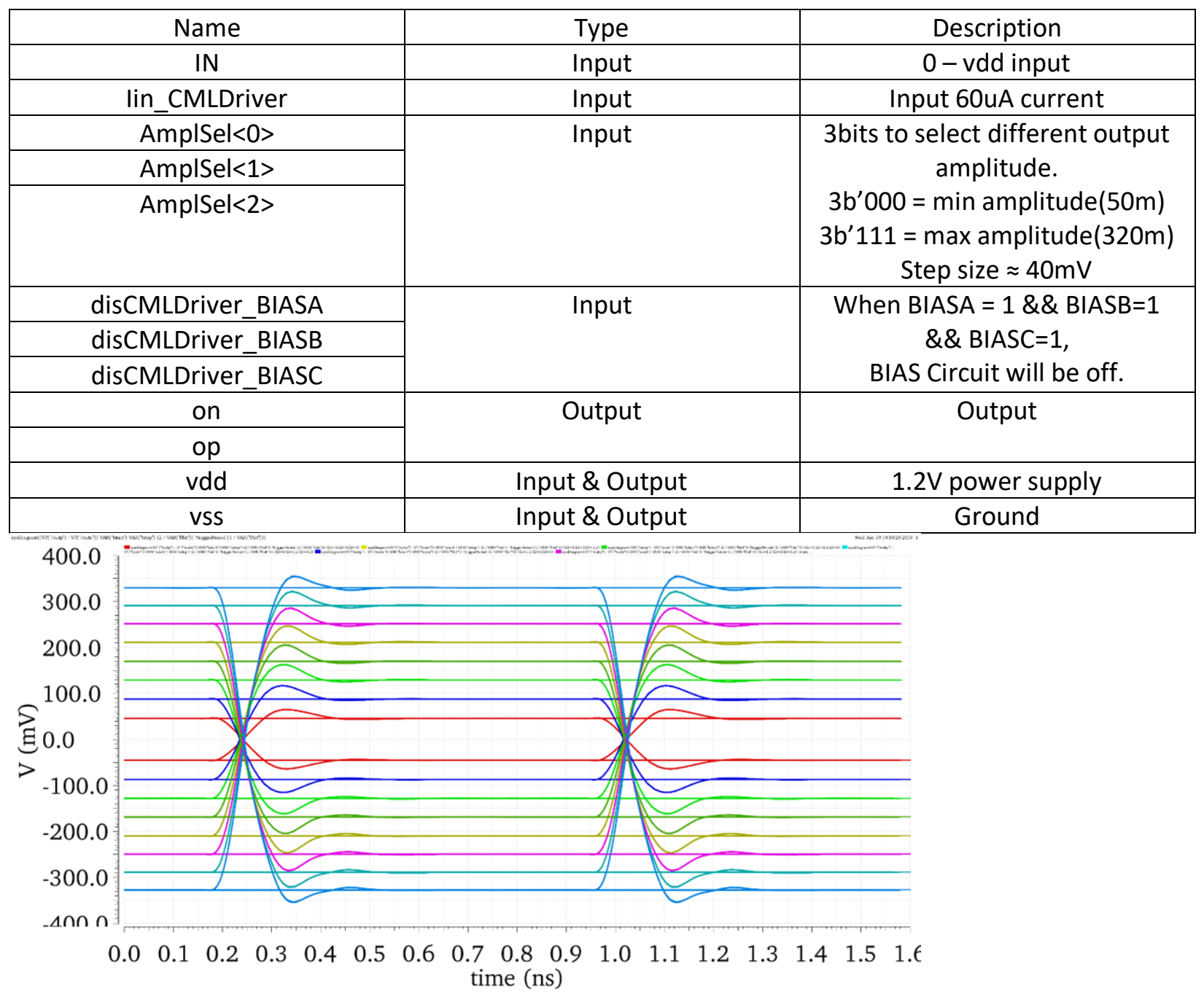

Figure 5.5.2 Post-layout Result of Tx

\subsection{ERX}

eRx is an IP block from IpGBT. It receives a differential signal and converts it into CMOS signal. 
FERMILAB-TM-2746-CMS-E

\begin{tabular}{|c|c|c|c|c|c|}
\hline Parameter & Description & Min & Nom & Max & Units \\
\hline $\mathrm{V}_{\mathrm{DD}}$ & Supply voltage range & 1.08 & 1.2 & 1.32 & $\mathrm{~V}$ \\
\hline$I_{D D}$ & Average current consumption & & 850 & & $\mu \mathrm{A}$ \\
\hline $\mathrm{V}_{\text {CMRX }}$ & Common-mode voltage range $^{A}$ & 70 & 600 & 1200 & $\mathrm{mV}$ \\
\hline $\mathrm{V}_{\mathrm{CM}}$ & Common-mode set voltage $^{B}$ & & $\mathrm{~V}_{\mathrm{DD}} / 2$ & & \\
\hline$\left|V_{I D}\right|$ & Differential voltagec & 140 & 200 & 400 & $\mathrm{mV}$ \\
\hline $\mathrm{V}_{\text {IDTH }}$ & Differential input ${ }^{\mathrm{C}}$ high threshold & & & 70 & $\mathrm{mV}$ \\
\hline $\mathrm{V}_{\text {IDTL }}$ & Differential input ${ }^{\mathrm{C}}$ low threshold & -70 & & & $\mathrm{mV}$ \\
\hline $\mathrm{V}_{\mathrm{IH}}$ & Single-ended input high voltage & & 700 & $\mathrm{~V}_{\mathrm{DD}}+200$ & $\mathrm{mV}$ \\
\hline $\mathrm{V}_{\mathrm{IL}}$ & Single-ended input low voltage & -40 & 500 & & $\mathrm{mV}$ \\
\hline$Z_{I D}$ & Differential input impedance & 80 & 100 & 125 & $\Omega$ \\
\hline$J_{R}$ & Random noise jitter & & & $10^{\mathrm{F}}$ & ps rms \\
\hline $\mathrm{J}_{\mathrm{PW}}$ & Pattern or pulse width dependent jitter ${ }^{D}$ & & & $10^{\mathrm{F}}$ & ps \\
\hline $\mathrm{T}_{\mathrm{R} / \mathrm{F}}$ & Output rise/fall time & & 30 & & ps \\
\hline PSR & Power supply rejection & & & $10^{\mathrm{F}}$ & $\mathrm{ps} / 100 \mathrm{mV}$ \\
\hline CMR & Common mode rejection & & & $10^{\mathrm{F}}$ & $\mathrm{ps} / 100 \mathrm{mV}$ \\
\hline $\mathrm{C}_{\mathrm{CM}}$ & Common mode termination capacitance & & tbd. & & $\mathrm{pF}$ \\
\hline$L_{0}$ & Output load & 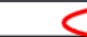 & 100 & & $\mathrm{fF}$ \\
\hline
\end{tabular}

A Common mode: $\left(\mathrm{V}_{\mathrm{DP}}+\mathrm{V}_{\mathrm{DN}}\right) / 2$

${ }^{B}$ For AC coupled signals

${ }^{\mathrm{C}}$ Differential voltage: $\mathrm{V}_{\mathrm{DP}}-\mathrm{V}_{\mathrm{DN}}$

${ }^{D}$ Change of delay through the receiver with PRBS $2^{12}-1 @ 1.28 \mathrm{Gbit} / \mathrm{s}$ or varying pulse width $\mathrm{T}_{\mathrm{PW}}>1 \mathrm{~ns}$

EFor LVDS: $400 \mathrm{mV}$

${ }^{\mathrm{F}}$ If $<=1 \mathrm{ps}$ possible (even with reduced input range), receiver could be used as hit receiver

Figure 5.6.1 Electrical specification of eRx

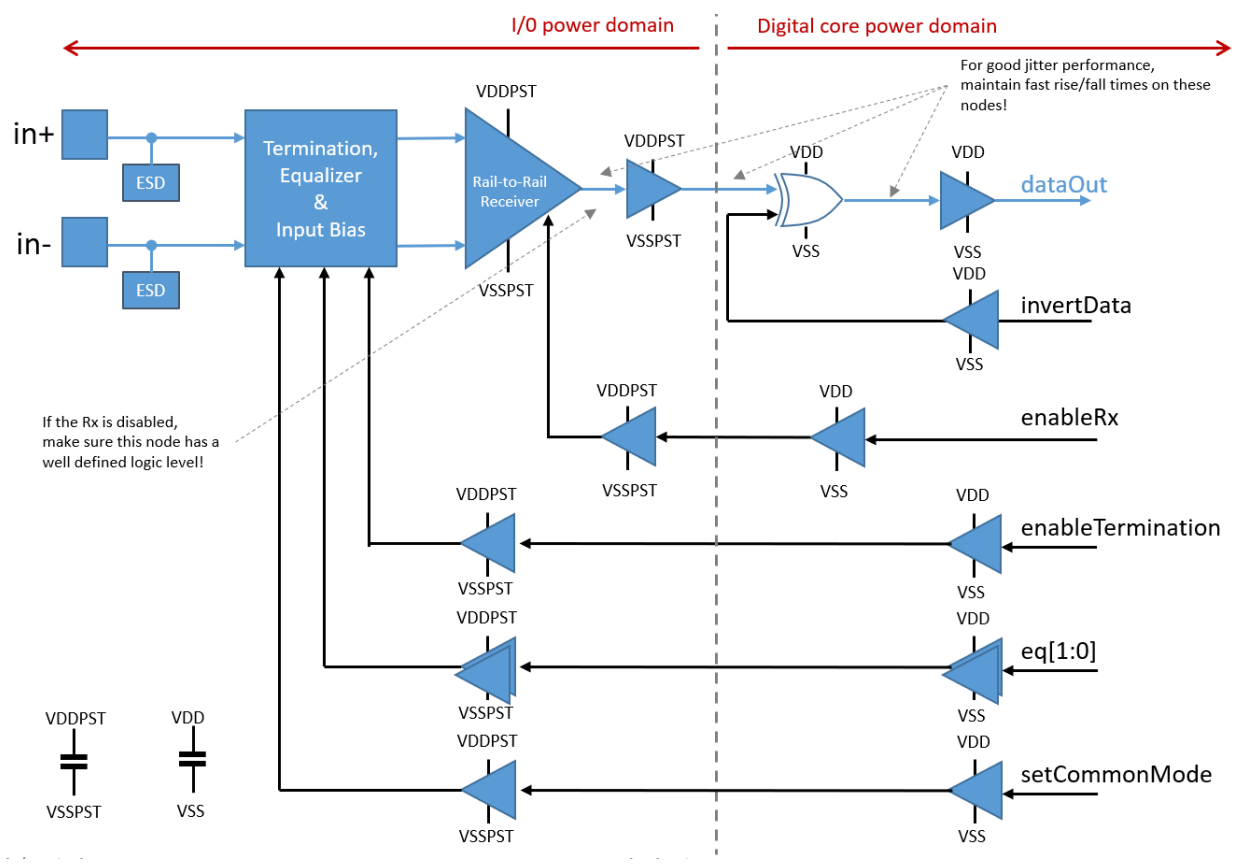

Figure 5.6.2 block diagram of eRx 


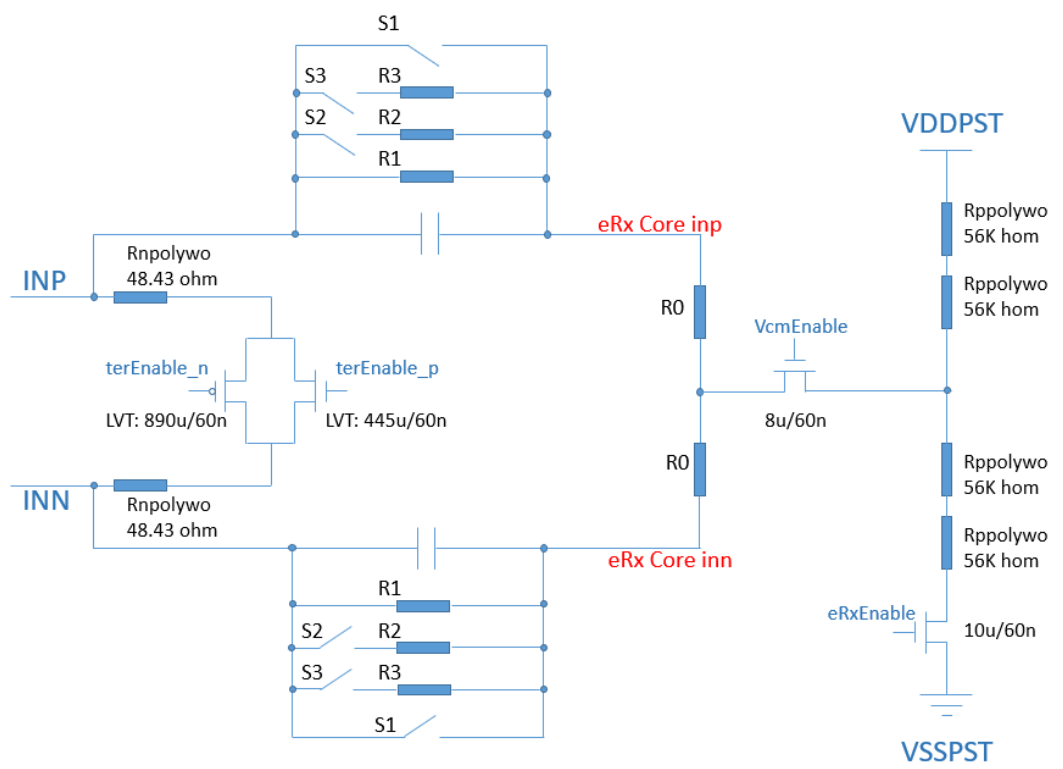

Figure 5.6.3 eRx input interface, $C=730 \mathrm{fC}, R 1=3.45 \mathrm{kOhm}, R 2=5.3 \mathrm{kOhm}, R 3=1.8 \mathrm{kOhm}$

Table 5.6.1 eRx block pinout

\begin{tabular}{|c|c|c|}
\hline Name & Type & Description \\
\hline enableRx & Input & Enable eRx, active high \\
\hline setCommonMode & Input & Set the input common mode voltage to vdd/2, active high \\
\hline enableTermination & Input & Enable the $100 \mathrm{Ohm}$ termination resistance, active high \\
\hline int, in- & $\begin{array}{l}\text { Differential } \\
\text { input }\end{array}$ & \\
\hline invertData & input & Invert the signal, active high \\
\hline Eq[1:0] & input & Internal equalization control \\
\hline dataOut & Output & CMOS output \\
\hline
\end{tabular}

Table 5.6.1 eRx equalization control

\begin{tabular}{|l|l|l|l|l|l|}
\hline Eq[1] & Eq[0] & S1 & S2 & S3 & \\
\hline $\mathbf{0}$ & $\mathbf{0}$ & 1 & $x$ & $x$ & $0 \Omega$ \\
\hline $\mathbf{0}$ & $\mathbf{1}$ & 0 & 0 & 1 & $\mathrm{R} 1 / / \mathrm{R} 3$ \\
\hline $\mathbf{1}$ & $\mathbf{0}$ & 0 & 1 & 0 & $\mathrm{R} 1 / / \mathrm{R} 2$ \\
\hline $\mathbf{1}$ & $\mathbf{1}$ & $\mathbf{0}$ & 0 & 0 & $\mathrm{R} 1$ \\
\hline
\end{tabular}




\subsection{CHARGE INJECTION}

The Charge injection block injects charge to the pre-amplifier for testability. It takes a step pulse control signal as input to generate the injected charge, and 5-bit input data to select injected charge, from $1 \mathrm{fC}$ ( $5^{\prime}$ b00000) to $32 \mathrm{fC}$ ( $5^{\prime}$ b11111). This block also can be tested with off-chip CSA to calibration injected charge.

The capacitor used to generate charge is $60 \mathrm{fC}$ in ETROC1.

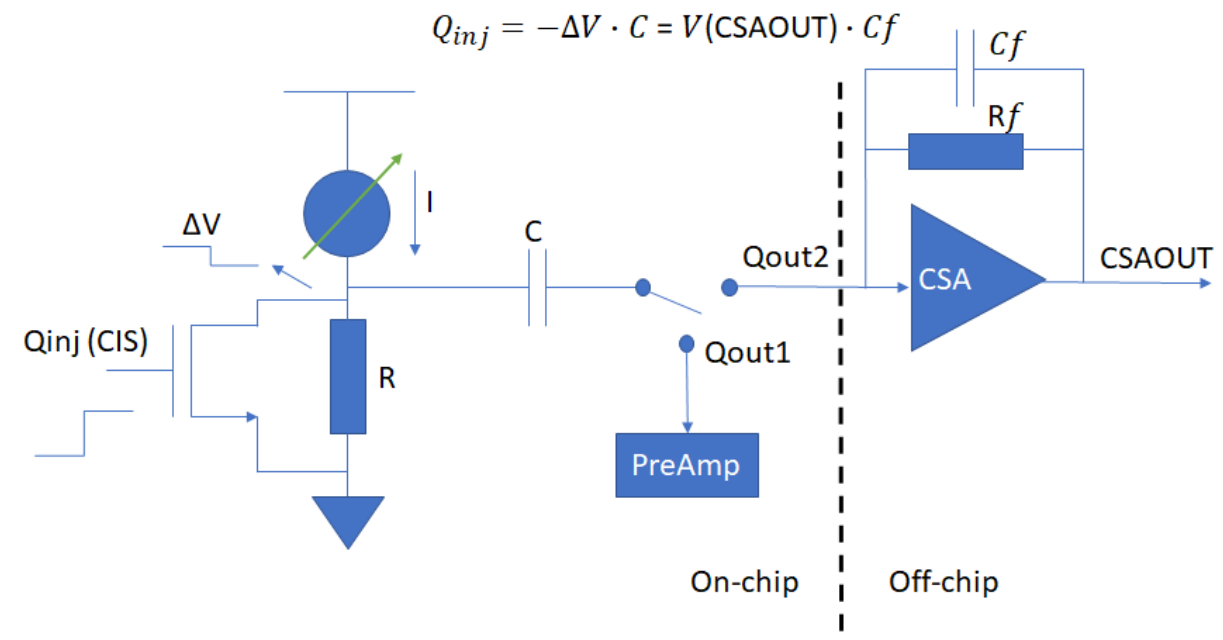

Figure 5.7.1 Block diagram of Charge Injection

Table 5.7 Charge Injection block pinout

\begin{tabular}{|c|c|c|c|}
\hline Num. & Pin name & Type & Description \\
\hline 1 & VREF & Analog input & Bias Voltage for Current Bias circuit \\
\hline 2 & $\mathrm{CIS}$ & Digital input & Charge injection step pulse control signal \\
\hline 3 & QSELL4:0> & Digital input & $\begin{array}{l}\text { Select injected charge, from } 1 \mathrm{fC}\left(5^{\prime} \text { b00000) to } 32 \mathrm{fC}\right. \\
\left(5^{\prime} \text { b11111) }\right. \\
\text { Typical charge is } 6 \mathrm{fC}\left(5^{\prime} \mathrm{b} 00101\right)\end{array}$ \\
\hline 4 & EN & Digital input & $\begin{array}{l}\text { Enable Charge Injection (onchip); } \\
\text { Set low with minimum power consumption }\end{array}$ \\
\hline 5 & QOUTSel & Digital input & $\begin{array}{l}\text { Select charge output trace (default high: on-chip PreAmp) } \\
\text { Output charge to off-chip CSA for testing when setting low. }\end{array}$ \\
\hline 6 & TV1 & Analog inout & Input voltage step pulse or output voltage for testing \\
\hline 7 & Qout1 & Analog output & Output signal to on-chip PreAmp \\
\hline 8 & Qout2 & Analog output & Output signal to off-chip CSA \\
\hline
\end{tabular}




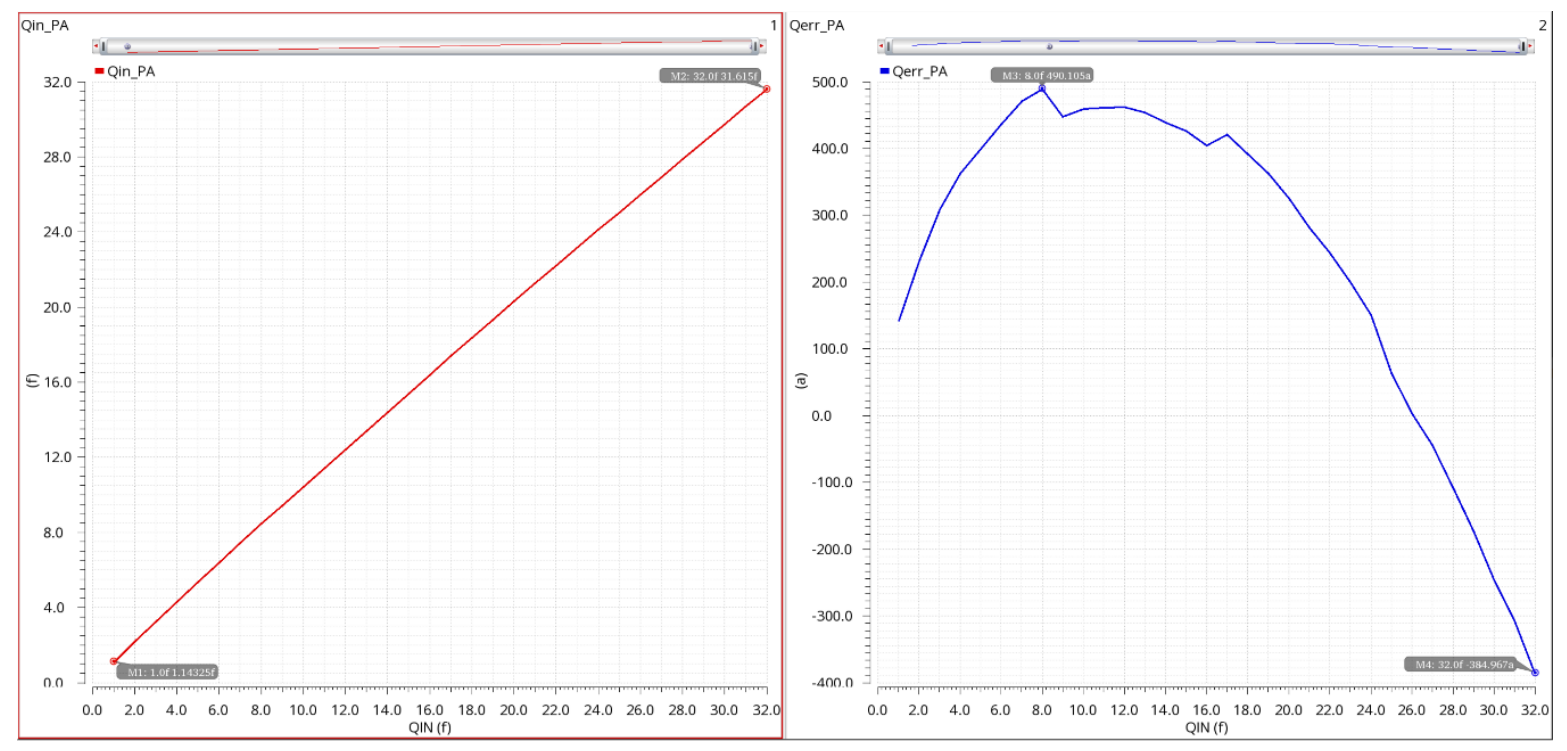

Figure 5.7.2 Simulated Injected charge vs input 5-bit control

\subsection{READOUT TEST PATTERN GENERATOR}

The purpose of readout test pattern generator (ROTestGen) is to generate test pattern for testing of SRO and DRO. ROTestGen outputs a configurable pattern so that the test pattern of each of 16 pixels could be different. The figure below shows a conceptual pixel block diagram including ROTestGen. A 4bit input is used to config output pattern in ROTestGen. ROTestGen shares 4-bit LSBs with DAC which is bypassed in readout test mode (TestRO==1'b1).

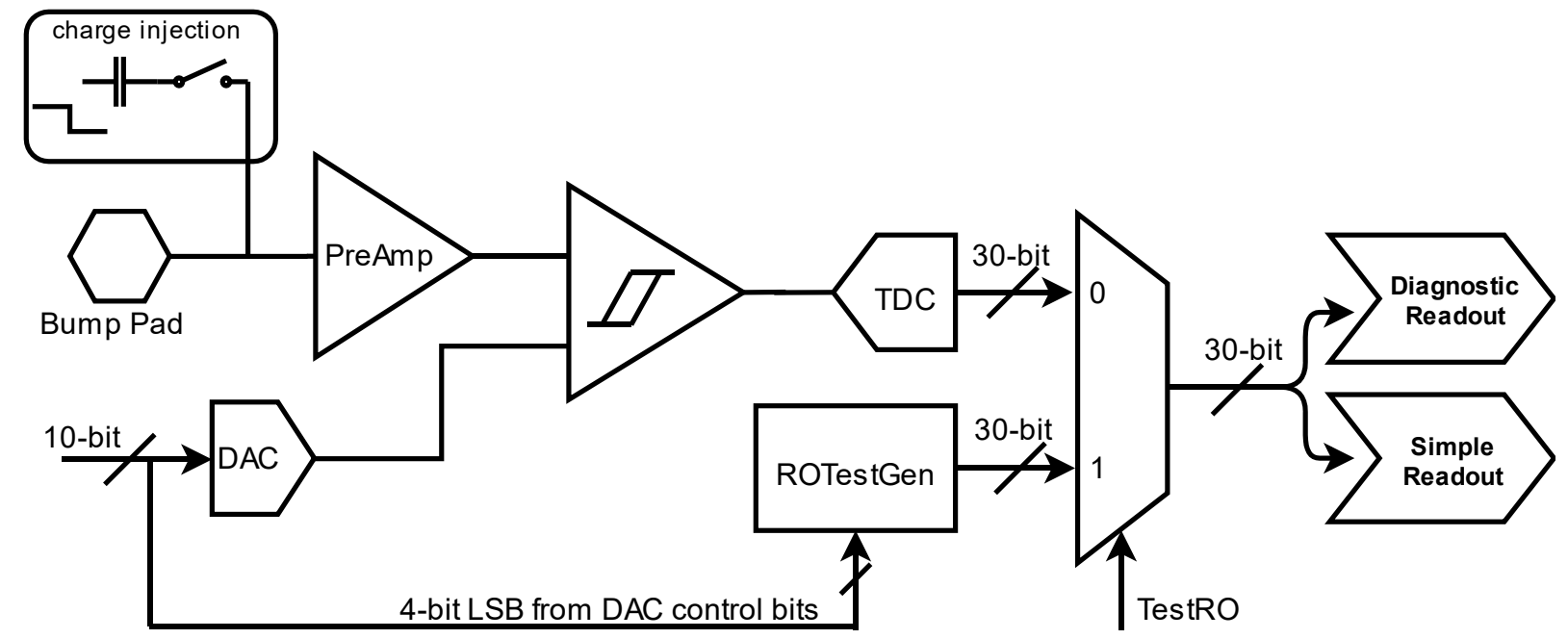

Figure 5.8.1 Block diagram of Readout test pattern generator 
In the actual implementation, ROTestGen and the following mux are implemented in a single block, which is called ROTestCtrl. The 16-bit LSBs output (30-bit output in total) of ROTestCtrl is generated from a 16-bit counter. At rising edge of the input clock, the counter increases one. The 4 configuration bits are concatenated to the 16 counter bits, and 10 fixed bits locates at MSBs. The TestRO connects to enableMon in TDC. The pinout of ROTestCtrl is listed below.

Table 5.8.1 ROTestGen block pinout

\begin{tabular}{|c|c|c|c|}
\hline Num. & Pin name & Type & Description \\
\hline 1 & CLK & Digital input & $40 \mathrm{MHz}$ clock \\
\hline 2 & CFGROTest $<3: 0>$ & Digital input & 4 configuration bits used to distinguish pixel. \\
\hline 3 & TestRO & Digital input & $\begin{array}{l}\text { Control of readout test mode. } \\
\text { When TestRO==1'b1, test pattern is sent for } \\
\text { readout. } \\
\text { When TestRO==1'b0, TDC output is sent for } \\
\text { readout. }\end{array}$ \\
\hline 4 & Dataln<29:0> & Digital input & 30 bits TDC output data \\
\hline 5 & DataOut<29:0> & Digital output & $\begin{array}{l}\text { When TestRO==1'b1, test pattern is sent for } \\
\text { readout. } \\
\text { DataOut }<15: 0>\text { is the 16-bit counter output. } \\
\text { DataOut }<19: 16>==\text { CFGROTest }<3: 0> \\
\text { DataOut }<29: 20>==10^{\prime} \text { b1010101010 } \\
\text { When TestRO==1'b0, TDC output is sent for } \\
\text { readout. }\end{array}$ \\
\hline
\end{tabular}

\section{DATA READOUT OF ETROC1}

Two readout schemes are implemented in ETROC1. They are named diagnostic mode readout and simple readout, respectively.

\subsection{DIAGNOSTIC MODE READOUT}

In diagnostic mode readout, one of 16 pixels is selected and the test pattern or the date from the TDC is readout through DMRO block continuously at 30-bit per clock period. Since DMRO block adds 2-bit overhead per 30-bit word, the total data rate is $1.28 \mathrm{Gbps}$. The $12 \mathrm{C}$ registers, DMRO_COL[3:0] and OE_DMRO_Row[3:0], can be used to select the pixel to be readout. 


\subsection{SIMPLE READOUT}

Simple readout is one of two readout schemes in ETROC1, through which the data stored in selected pixels(pixel) can be read out as a data frame with a predefined header and trailer. Each pixel includes a RAM which stores data from the TDC with width of 30-bit and depth of 256 . Four pixels in a column share a bus, and four column-level buses connect to a module called SRO controller (simple readout controller). When normal running, $W E=1$, address increments, TDC values or test pattern (see test pattern generator section) are stored into the RAM buffers. When L1ACC occurs, WE is dropped (no data can be written into RAM), SRO controller sends the header word (SOF, start of frame) and then selects first enabled pixel. The address loops once reading out entire RAM buffer. Then it moves on to next enabled pixel, dumping out the RAM buffer. When the last word of the last enabled pixel is sent, the frame is ended up with the trailer word (EOF, end of frame). It returns to capture mode with WE $=1$. The simple readout shares the same DMRO block and the pads with diagnostic mode readout.

The I2C register, $\mathrm{ROI}$ (region of interest), specifies which pixels are enabled for readout. The highest index pixels will read out first. E.g. ROI=16'h9201 will cause pixels 15 (Row3Col3), 12 (Row3Col0), 9 (Row2Col1), and 0 (Row0Col0) readout in that order.

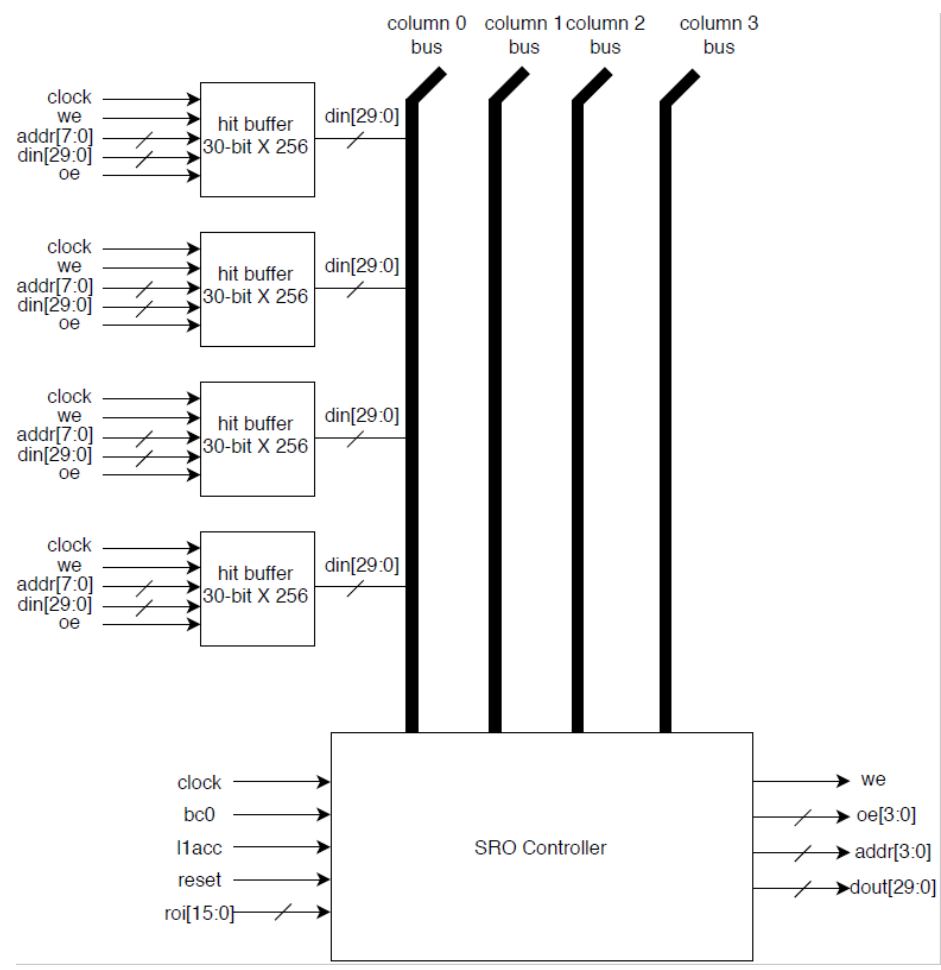

Figure 6.2.1 Block diagram of simple readout

\begin{tabular}{|c|c|c|c|}
\hline 18'h25555 & 12-bit L1ACC_ID & 0-bit to $16 \times 256 \times 30$-bit Frame Body. \\
\cline { 1 - 1 } Length depends on ROI
\end{tabular}

Figure 6.2.2 frame format of simple readout 


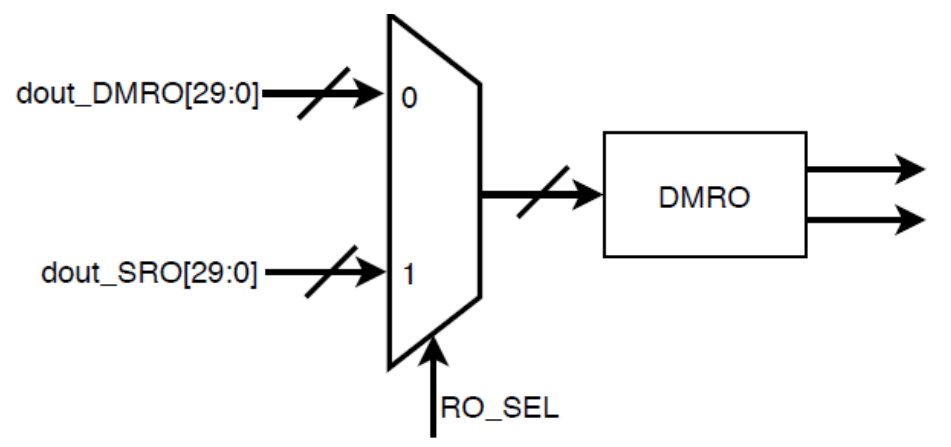

Figure 6.2.2 Block diagram of data path shared by two readout schemes

\begin{tabular}{|c|c|c|c|}
\hline \multicolumn{5}{|c|}{ TDCData[29:0] } \\
\hline TOT[8:0] & TOA[9:0] & Cal[9:0] & hitFlag \\
\hline
\end{tabular}

(a)

\begin{tabular}{|c|c|c|c|}
\hline \multicolumn{4}{|c|}{ TDCData[29:0] } \\
\hline hitFlag & Cal[9:0] & TOA[9:0] & TOT[8:0] \\
\hline
\end{tabular}

(b)

Figure 6.2.3 data format of 30-bit word from TDC: (a) data format of standalone pixel and pixel array, (b) data format of TDC test chip

\begin{tabular}{|c|c|c|c|}
\hline $\begin{array}{c}P_{-} \mathbf{0} 0 \\
\text { index: } 0\end{array}$ & $\begin{array}{c}\text { P_0_1 } \\
\text { index: } 4\end{array}$ & $\begin{array}{c}\text { P_0_2 } \\
\text { index: } 8\end{array}$ & $\begin{array}{c}\text { P_0_3 } \\
\text { index: } 12\end{array}$ \\
\hline $\begin{array}{c}P_{-} \text {1_0 } \\
\text { index: } 1\end{array}$ & $\begin{array}{c}P_{-1} 1 \_1 \\
\text { index: } 5\end{array}$ & $\begin{array}{c}P_{-1} 1 \_2 \\
\text { index: } 8\end{array}$ & $\begin{array}{c}\text { P_1_3 } \\
\text { index: } 13\end{array}$ \\
\hline $\begin{array}{c}\text { P_2_0 } \\
\text { index: } 2\end{array}$ & $\begin{array}{c}\text { P_2_1 } \\
\text { index: } 6\end{array}$ & $\begin{array}{c}\text { P_2_2 } \\
\text { index: } 10\end{array}$ & $\begin{array}{c}\text { P_2_3 } \\
\text { index: } 14\end{array}$ \\
\hline $\begin{array}{c}\text { P_3_0 } \\
\text { index: } 3\end{array}$ & $\begin{array}{c}\text { P_3_1 } \\
\text { index: } 7\end{array}$ & $\begin{array}{c}\text { P_3_2 } \\
\text { index: } 11\end{array}$ & $\begin{array}{c}\text { P_3_3 } \\
\text { index: } 15\end{array}$ \\
\hline \multicolumn{4}{|c|}{ Chip peripherals } \\
\hline
\end{tabular}

Figure 6.2.4 pixels map 
FERMILAB-TM-2746-CMS-E

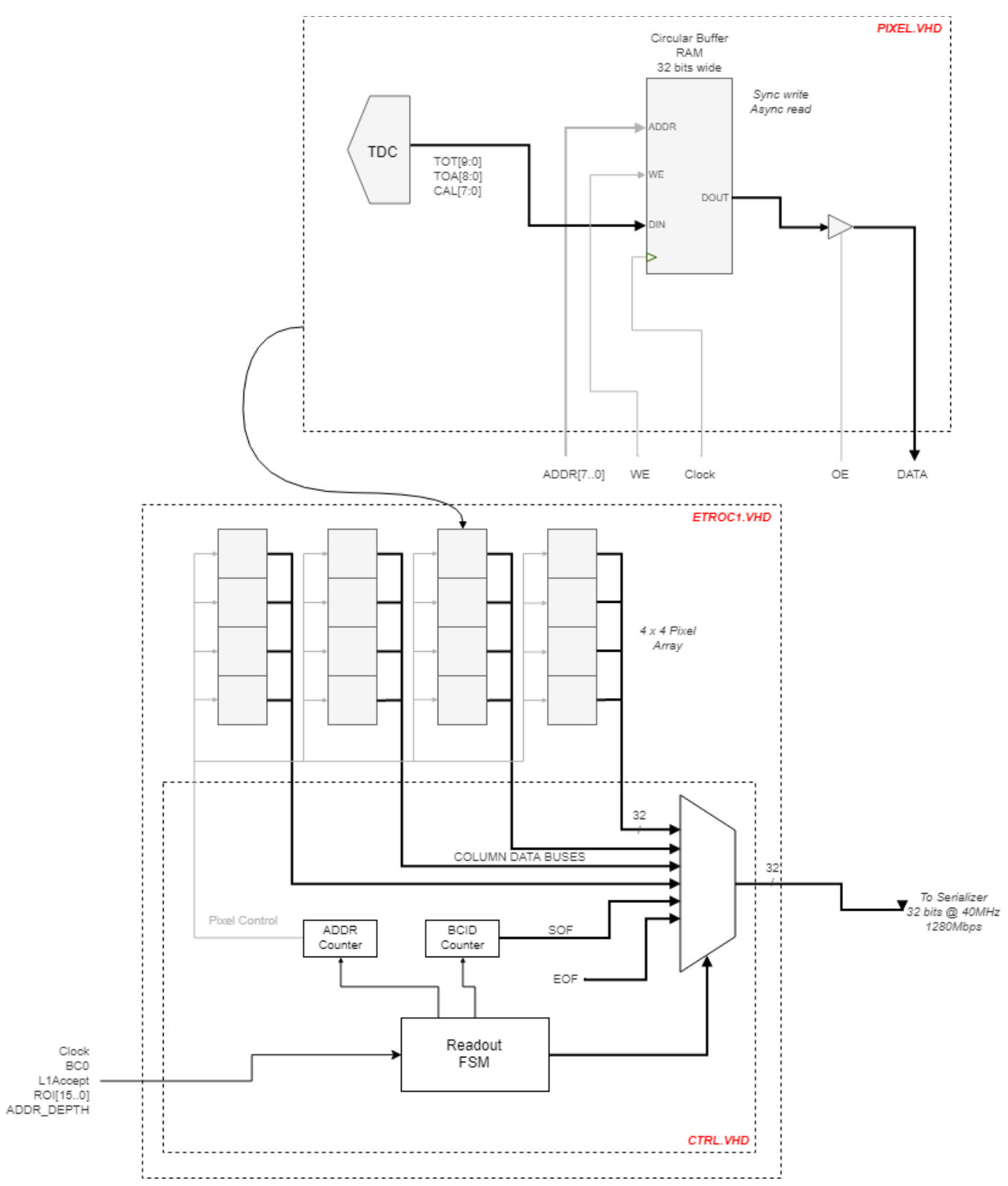

Figure 6.2.5 detailed block diagram of simple readout. 32-bit words have been changed to 30-bit to match with DMRO block. A $12-$ bit BCID counter increments as clock goes and can be reset when bcO occurs. The instant BCID value will become part of SOF as L1ACC_ID when L1ACC occurs. 


\section{FERMILAB-TM-2746-CMS-E}

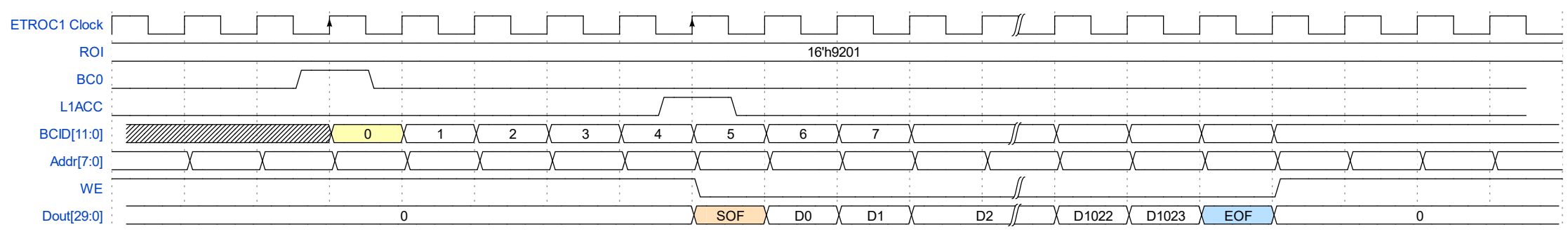

Figure 6.2.5 an example of simple readout sequence. In this case, the L1ACC_ID is 4 and the frame length is 1026 words including the SOF and EOF. 